\title{
Hybrid Approach with Active Set Identification for Mathematical Programs with Complementarity Constraints *
}

\author{
Gui-Hua $\operatorname{Lin}^{\dagger}$ and Masao Fukushima ${ }^{\ddagger}$
}

January 2003, Revised June 2004

\begin{abstract}
We consider a mathematical program with complementarity constraints (MPCC). Our purpose is to develop methods that enable us to compute a solution or a point with some kind of stationarity to MPCC by solving a finite number of nonlinear programs. We apply an active set identification technique to a smoothing continuation method (Fukushima and Pang, 1999) and propose a hybrid algorithm for solving MPCC. We also develop two kinds of modifications, one of which makes use of an index addition strategy and the other adopts an index subtraction strategy. We show that, under reasonable assumptions, all the proposed algorithms possess a finite termination property. Further discussions and computational results are given as well.

Key Words. mathematical program with complementarity constraints, MPCC-LICQ, weak secondorder necessary condition, (B-, M-, C-) stationarity, asymptotically weak nondegeneracy, identification function.
\end{abstract}

\section{Introduction}

Mathematical programs with equilibrium constraints (MPECs) have been receiving much attention in the optimization community. There are many practical problems in engineering and economics that can be modelled as MPECs. MPECs also find applications in mathematical programming itself, for example, through reformulation of bilevel programming. For further applications, see the monograph [20] and the references therein.

In this paper, we consider the following mathematical program with complementarity constraints (MPCC), which constitutes an important subclass of MPECs:

$$
\begin{array}{cl}
\min & f(z) \\
\text { s.t. } & g(z) \leq 0, h(z)=0 \\
& G(z) \geq 0, H(z) \geq 0 \\
& G(z)^{T} H(z)=0,
\end{array}
$$

where $f: \Re^{n} \rightarrow \Re, g: \Re^{n} \rightarrow \Re^{p}, h: \Re^{n} \rightarrow \Re^{q}$, and $G, H: \Re^{n} \rightarrow \Re^{m}$ are all twice continuously differentiable functions. The major difficulty in solving problem $(\mathrm{P})$ is that its constraints

\footnotetext{
${ }^{*}$ This work was supported in part by the Scientific Research Grant-in-Aid from the Ministry of Education, Science, Sports, and Culture of Japan.

${ }^{\dagger}$ Department of Applied Mathematics, Dalian University of Technology, Dalian 116024, China./ Department of Applied Mathematics and Physics, Graduate School of Informatics, Kyoto University, Kyoto 606-8501, Japan. E-mail: ghlin@amp.i.kyoto-u.ac.jp.

${ }^{\ddagger}$ Department of Applied Mathematics and Physics, Graduate School of Informatics, Kyoto University, Kyoto 606-8501, Japan. E-mail: fuku@amp.i.kyoto-u.ac.jp.
} 
fail to satisfy a standard constraint qualification at any feasible point [4], so that standard methods are not guaranteed to solve this problem. There have been proposed several approaches such as sequential quadratic programming approach $[9,16,20]$, implicit programming approach $[3,20]$, penalty function approach $[12,13,19,20]$, active-set approach [11], and reformulation approach [7, 10, 17, 18, 22]. In particular, Fukushima and Pang [10] considered a smoothing continuation method and showed, under the MPCC-linear independence constraint qualification and an additional condition called the asymptotically weak nondegeneracy, that an accumulation point of KKT points satisfying the weak second-order necessary conditions for the perturbed problems is a B-stationary point of the original problem. Subsequently, similar schemes were presented by Scholtes [22] and Lin and Fukushima [17, 18]. However, these methods require to solve an infinite sequence of nonlinear programs. The purpose of this paper is to develop methods that enable us to compute a solution or a point with some kind of stationarity to problem $(\mathrm{P})$ by solving a finite number of nonlinear programs.

The rest of the paper is organized as follows. In the next section, we recall some basic concepts for problem (P) and ordinary nonlinear programming problems. In Section 3, we first introduce the smoothing continuation method [10] and then, we apply an active set identification technique to the method and present our hybrid algorithms. A comprehensive convergence analysis is also included. We make some remarks in Section 4 and report our numerical experience in Section 5. Throughout the paper, we let $\mathcal{F}$ denote the feasible region of problem $(\mathrm{P})$ and, for a function $F: \Re^{n} \rightarrow \Re^{m}$ and a given vector $z \in \Re^{n}$, let

$$
\mathcal{I}_{F}(z):=\left\{i \mid F_{i}(z)=0\right\}
$$

stand for the active index set of $F$ at $z$.

\section{Preliminaries}

In this section, we state some basic concepts for MPCC and ordinary nonlinear programming problems. We first consider the standard nonlinear programming problem

$$
\begin{array}{cl}
\min & f(z) \\
\text { s.t. } & c_{i}(z) \leq 0, \quad i=1, \cdots, l, \\
& c_{i}(z)=0, \quad i=l+1, \cdots, s,
\end{array}
$$

where $f: \Re^{n} \rightarrow \Re$ and $c: \Re^{n} \rightarrow \Re^{s}$ are twice continuously differentiable.

Definition 2.1 We say $z$ to be a stationary point of problem (2.1) if it is feasible to (2.1) and there exists a Lagrange multiplier vector $\lambda \in \Re^{s}$ such that

$$
\begin{aligned}
& \nabla f(z)+\nabla c(z) \lambda=0, \\
& \lambda_{i} \geq 0, \lambda_{i} c_{i}(z)=0, \quad i=1, \cdots, l .
\end{aligned}
$$

Definition 2.2 Let $z$ be a stationary point of problem (2.1) and $\lambda$ be a Lagrange multiplier vector corresponding to $z$. We say the weak second-order necessary condition (WSONC) holds at $z$ if we have

$$
d^{T}\left(\nabla^{2} f(z)+\sum_{i=1}^{s} \lambda_{i} \nabla^{2} c_{i}(z)\right) d \geq 0
$$


for any $d \in \mathcal{T}(z):=\left\{d \in \Re^{n} \mid d^{T} \nabla c_{i}(z)=0, \quad \forall i \in \mathcal{I}_{c}(z)\right\}$.

Next, we recall some concepts related to problem $(\mathrm{P})$.

Definition 2.3 The MPCC-linear independence constraint qualification (MPCC-LICQ) is said to hold at $\bar{z} \in \mathcal{F}$ if the set of vectors

$$
\begin{aligned}
& \left\{\nabla g_{l}(\bar{z}), \nabla h_{r}(\bar{z}), \nabla G_{i}(\bar{z}), \nabla H_{j}(\bar{z}) \mid\right. \\
& \left.l \in \mathcal{I}_{g}(\bar{z}), r=1, \cdots, q, i \in \mathcal{I}_{G}(\bar{z}), j \in \mathcal{I}_{H}(\bar{z})\right\}
\end{aligned}
$$

is linearly independent.

This condition is not particularly stringent [23] and has been assumed often in the literature on MPCCs $[10,12,13,17,22]$. Note that this definition is different from the standard definition of LICQ in nonlinear programming theory that would require the gradient of the function $G(z)^{T} H(z)$ be linearly independent of the above vectors, which cannot happen in any case actually.

In the study of MPCCs, there are several kinds of stationarity defined for problem (P).

Definition 2.4 We say $\bar{z} \in \mathcal{F}$ is a Bouligand or B-stationary point of problem $(\mathrm{P})$ if it satisfies

$$
d^{T} \nabla f(\bar{z}) \geq 0, \quad \forall d \in \mathcal{T}(\bar{z}, \mathcal{F}),
$$

where

$$
\mathcal{T}(\bar{z}, \mathcal{F}):=\left\{d \in \Re^{n} \mid t_{k}\left(z^{k}-\bar{z}\right) \rightarrow d, z^{k} \rightarrow \bar{z}, z^{k} \in \mathcal{F}, t_{k} \geq 0, k=1,2, \cdots\right\}
$$

stands for the tangent cone of $\mathcal{F}$ at $\bar{z}$.

Definition 2.5 [21] (1) A vector $\bar{z} \in \mathcal{F}$ is said to be a Clarke or C-stationary point of (P) if there exist multiplier vectors $\bar{\lambda}_{g} \in \Re^{p}, \bar{\lambda}_{h} \in \Re^{q}$, and $\bar{\lambda}_{G}, \bar{\lambda}_{H} \in \Re^{m}$ such that

$$
\begin{aligned}
& \nabla f(\bar{z})+\nabla g(\bar{z}) \bar{\lambda}_{g}+\nabla h(\bar{z}) \bar{\lambda}_{h}-\nabla G(\bar{z}) \bar{\lambda}_{G}-\nabla H(\bar{z}) \bar{\lambda}_{H}=0, \\
& \bar{\lambda}_{g} \geq 0, \quad \bar{\lambda}_{g}^{T} g(\bar{z})=0, \\
& \bar{\lambda}_{G, i}=0, \quad i \notin \mathcal{I}_{G}(\bar{z}), \\
& \bar{\lambda}_{H, i}=0, \quad i \notin \mathcal{I}_{H}(\bar{z}), \\
& \bar{\lambda}_{G, i} \bar{\lambda}_{H, i} \geq 0, \quad \forall i \in \mathcal{I}_{G}(\bar{z}) \cap \mathcal{I}_{H}(\bar{z}) .
\end{aligned}
$$

(2) $\bar{z} \in \mathcal{F}$ is called a Mordukhovich or M-stationary point of (P) if there exist multiplier vectors $\bar{\lambda}_{g} \in \Re^{p}, \bar{\lambda}_{h} \in \Re^{q}, \bar{\lambda}_{G}, \bar{\lambda}_{H} \in \Re^{m}$ such that (2.3)-(2.6) hold and

$$
\text { either } \bar{\lambda}_{G, i}>0, \bar{\lambda}_{H, i}>0 \text { or } \bar{\lambda}_{G, i} \bar{\lambda}_{H, i}=0, \quad \forall i \in \mathcal{I}_{G}(\bar{z}) \cap \mathcal{I}_{H}(\bar{z}) \text {. }
$$

In order to get a desirable stationary point of $(\mathrm{P})$, some additional conditions need to be assumed. Here, we introduce one of them.

Definition 2.6 Let $\bar{z} \in \mathcal{F}$. If there exist multiplier vectors $\bar{\lambda}_{g}, \bar{\lambda}_{h}, \bar{\lambda}_{G}$, and $\bar{\lambda}_{H}$ satisfying $(2.3)-$ (2.6) and

$$
\bar{\lambda}_{G, i} \bar{\lambda}_{H, i} \neq 0, \quad i \in \mathcal{I}_{G}(\bar{z}) \cap \mathcal{I}_{H}(\bar{z}),
$$

we say that the upper-level strict complementarity (ULSC) condition holds at $\bar{z}$.

It is obvious that any M-stationary point satisfying the ULSC condition must be B-stationary to problem $(\mathrm{P})$. 


\section{Hybrid Algorithms for MPCC}

As mentioned earlier, the aim of this paper is to develop methods that enable us to compute a solution or some kind of stationary points of problem $(\mathrm{P})$ by solving a finite sequence of nonlinear programs, unlike many existing methods that require to solve an infinite sequence of nonlinear programs. To this end, we apply an active set identification technique to a smoothing continuation method [10] and present some hybrid algorithms in this section. Further discussions and some extensions will be given in Section 4 .

\subsection{Smoothing continuation method for MPCC}

Consider the smoothing continuation method [10] that uses the perturbed Fischer-Burmeister function

$$
\phi_{\epsilon}(a, b):=a+b-\sqrt{a^{2}+b^{2}+2 \epsilon^{2}},
$$

where $\epsilon \geq 0$. Define the function $\Phi_{\epsilon}: \Re^{n} \rightarrow \Re^{m}$ by

$$
\Phi_{\epsilon}(z):=\left(\phi_{\epsilon}\left(G_{1}(z), H_{1}(z)\right), \cdots, \phi_{\epsilon}\left(G_{m}(z), H_{m}(z)\right)\right)^{T}
$$

and consider the nonlinear programming problem

$$
\begin{array}{cl}
\min & f(z) \\
\text { s.t. } & \Phi_{\epsilon}(z)=0, \\
& g(z) \leq 0, h(z)=0 .
\end{array}
$$

Note that $\left(P_{0}\right)$ is equivalent to problem $(\mathrm{P})$ and $\Phi_{\epsilon}$ is differentiable everywhere for any $\epsilon>0$. We assume that problem $\left(P_{\epsilon}\right)$ has a solution (or a stationary point) $z^{\epsilon}$ for each small scalar $\epsilon>0$. We may expect to find a solution or a point with some kind of stationarity to problem (P) by tracing the trajectory $\left\{z^{\epsilon}\right\}$ as $\epsilon \rightarrow 0$. Suppose that $\left\{\epsilon_{k}\right\}$ is a positive sequence converging to zero. The following convergence result is given in [10].

Theorem 3.1 Let $z^{k}$ be a stationary point of problem $\left(P_{\epsilon_{k}}\right)$ and the sequence $\left\{z^{k}\right\}$ converge to $z^{*}$ as $\epsilon_{k} \rightarrow 0$. Suppose that the WSONC holds at each $z^{k}$, the MPCC-LICQ holds at $z^{*}$, and $\left\{z^{k}\right\}$ is asymptotically weakly nondegenerate. Then $z^{*}$ is B-stationary to problem $(P)$.

We now recall the asymptotically weak nondegeneracy [10], which is assumed in the above theorem and will also be employed in the subsequent analysis. Suppose $\left\{z^{k}\right\}$ converges to $z^{*}$ as $\epsilon_{k} \rightarrow 0$. Then $z^{*} \in \mathcal{F}$. It can be shown [10] that, for each $i \in \mathcal{I}_{G}\left(z^{*}\right) \cap \mathcal{I}_{H}\left(z^{*}\right)$,

$$
\nabla \Phi_{\epsilon_{k}, i}\left(z^{k}\right)=\frac{H_{i}\left(z^{k}\right)}{G_{i}\left(z^{k}\right)+H_{i}\left(z^{k}\right)} \nabla G_{i}\left(z^{k}\right)+\frac{G_{i}\left(z^{k}\right)}{G_{i}\left(z^{k}\right)+H_{i}\left(z^{k}\right)} \nabla H_{i}\left(z^{k}\right) .
$$

Therefore, every accumulation point $r$ of $\left\{\nabla \Phi_{\epsilon_{k}, i}\left(z^{k}\right)\right\}$ can be represented as

$$
r=\xi_{i}(r) \nabla G_{i}\left(z^{*}\right)+\eta_{i}(r) \nabla H_{i}\left(z^{*}\right)
$$

for some $\left(\xi_{i}(r), \eta_{i}(r)\right)$ with $\left(1-\xi_{i}(r)\right)^{2}+\left(1-\eta_{i}(r)\right)^{2} \leq 1$. We say that $\left\{z^{k}\right\}$ is asymptotically weakly nondegenerate if, for each $i \in \mathcal{I}_{G}\left(z^{*}\right) \cap \mathcal{I}_{H}\left(z^{*}\right)$, neither $\xi_{i}(r)$ nor $\eta_{i}(r)$ vanishes for any accumulation point $r$ of $\left\{\nabla \Phi_{\epsilon_{k}, i}\left(z^{k}\right)\right\}$. 
Roughly speaking, the asymptotically weak nondegeneracy of $\left\{z^{k}\right\}$ means that, for each $i \in \mathcal{I}_{G}\left(z^{*}\right) \cap \mathcal{I}_{H}\left(z^{*}\right), G_{i}\left(z^{k}\right)$ and $H_{i}\left(z^{k}\right)$ approach zero in the same order of magnitude. This property is obviously weaker than the nondegeneracy (lower-level strict complementarity), because it vacuously holds when $z^{*}$ is nondegenerate. It is even weaker than the upper-level strict complementarity (ULSC) condition, which is often employed in the literature on MPCC, see $[12,13,17,18,22]$.

In addition, Lin and Fukushima $[17,18]$ have established some new convergence results for their relaxation methods for MPCC. We can prove that the smoothing continuation method possesses similar convergence properties. Here, we state one of such results.

Theorem 3.2 Let $z^{k}$ be a stationary point of problem $\left(P_{\epsilon_{k}}\right)$ and, for each $k,\left(\lambda_{g}^{k}, \lambda_{h}^{k}, \lambda_{\Phi}^{k}\right)$ be a multiplier vector corresponding to $z^{k}$. Suppose that the sequence $\left\{z^{k}\right\}$ converges to $z^{*}$ as $\epsilon_{k} \rightarrow 0$ and, for each $k, \nabla_{z}^{2} L_{\epsilon_{k}}\left(z^{k}, \lambda_{g}^{k}, \lambda_{h}^{k}, \lambda_{\Phi}^{k}\right)$ is bounded below with constant $\alpha_{k} \geq 0$ on the corresponding tangent space $\mathcal{T}_{\varepsilon_{k}}\left(z^{k}\right)$, which means

$$
d^{T} \nabla_{z}^{2} L_{\epsilon_{k}}\left(z^{k}, \lambda_{g}^{k}, \lambda_{h}^{k}, \lambda_{\Phi}^{k}\right) d \geq-\alpha_{k}\|d\|^{2}, \quad \forall d \in \mathcal{T}_{\epsilon_{k}}\left(z^{k}\right),
$$

where

$$
\begin{aligned}
& L_{\epsilon}\left(z, \lambda_{g}, \lambda_{h}, \lambda_{\Phi}\right):= f(z)+\lambda_{g}^{T} g(z)+\lambda_{h}^{T} h(z)+\lambda_{\Phi}^{T} \Phi_{\epsilon}(z), \\
& \mathcal{T}_{\epsilon}(z):=\left\{d \in \Re^{n} \mid d^{T} \nabla \Phi_{\epsilon, i}(z)=0,\right. i=1, \cdots, m ; \\
& d^{T} \nabla g_{l}(z)=0, \quad l \in \mathcal{I}_{g}(z) ; \\
&\left.d^{T} \nabla h_{r}(z)=0, \quad r=1, \cdots, q\right\} .
\end{aligned}
$$

If the sequence $\left\{\alpha_{k}\right\}$ is bounded, $\left\{z^{k}\right\}$ is asymptotically weakly nondegenerate, and the MPCC$L I C Q$ holds at $z^{*}$, then $z^{*}$ is a B-stationary point of problem $(P)$.

Actually, the condition that problem $\left(P_{\epsilon_{k}}\right)$ satisfies the WSONC at $z^{k}$ for each $k$ means that $\nabla_{z}^{2} L_{\epsilon_{k}}\left(z^{k}, \lambda_{g}^{k}, \lambda_{h}^{k}, \lambda_{\Phi}^{k}\right)$ is bounded below with constant 0 . In consequence, Theorem 3.1 is actually a corollary of Theorem 3.2. Note that, for the matrix $\nabla_{z}^{2} L_{\epsilon_{k}}\left(z^{k}, \lambda_{g}^{k}, \lambda_{h}^{k}, \lambda_{\Phi}^{k}\right)$, there must exist a number $\alpha_{k}$ such that (3.3) holds. For example, any nonnegative scalar $\alpha_{k}$ such that $\left(-\alpha_{k}\right)$ is less than the smallest eigenvalue of $\nabla_{z}^{2} L_{\epsilon_{k}}\left(z^{k}, \lambda_{g}^{k}, \lambda_{h}^{k}, \lambda_{\Phi}^{k}\right)$ must satisfy (3.3). However, the WSONC means that the matrix should have some kind of semi-definiteness on the tangent space $\mathcal{T}_{\epsilon_{k}}\left(z^{k}\right)$. Therefore, the assumptions in Theorem 3.2 are weaker than the conditions of Theorem 3.1. Since the proof of Theorem 3.2 is similar to that of Theorem 3.1, it is omitted here, see [10].

\subsection{A hybrid algorithm for MPCC}

We first introduce an active set identification technique for MPCC. Active set identification plays an important role in optimization theory $[1,2,5,6,25]$. Accurate identification of active constraints is important from both theoretical and practical points of view. For problem $(\mathrm{P})$, by means of active set identification, the combinatorial constraints

$$
G(z) \geq 0, \quad H(z) \geq 0, \quad G(z)^{T} H(z)=0
$$

may be replaced by some equality and/or inequality constraints that are easier to deal with. 
For a point $\bar{z} \in \mathcal{F}$, let $\alpha(\bar{z}), \beta(\bar{z})$ and $\gamma(\bar{z})$ be the index sets defined by

$$
\begin{aligned}
\alpha(\bar{z}):=\left\{i \mid G_{i}(\bar{z})>0, H_{i}(\bar{z})=0\right\}, \\
\beta(\bar{z}):=\left\{i \mid G_{i}(\bar{z})=0, H_{i}(\bar{z})=0\right\}, \\
\gamma(\bar{z}):=\left\{i \mid G_{i}(\bar{z})=0, H_{i}(\bar{z})>0\right\},
\end{aligned}
$$

respectively. Obviously, $\alpha(\bar{z}) \cup \beta(\bar{z}) \cup \gamma(\bar{z})=\{1, \cdots, m\}$ and these index sets are mutually disjoint. We call $\bar{z}$ nondegenerate if $\beta(\bar{z})=\emptyset$ and degenerate if $\beta(\bar{z}) \neq \emptyset$. Let a sequence $\left\{z^{k}\right\}$ be generated so that it converges to $z^{*} \in \mathcal{F}$. If $z^{*}$ is nondegenerate, it is generally not difficult to identify the correct index sets finitely. However, when $z^{*}$ is degenerate, it is not necessarily easy to identify the active index sets. In the following, we will particularly be interested in the case where $\left\{z^{k}\right\}$ is convergent to a degenerate point $z^{*} \in \mathcal{F}$.

Let $\left\{\epsilon_{k}\right\}$ be a positive sequence converging to zero and let $z^{k}$ stand for a solution or a stationary point of problem $\left(P_{\epsilon_{k}}\right)$ for each $k$. Suppose that $\left\{z^{k}\right\}$ converges to some $z^{*}$ throughout Section 3. Note that

$$
G_{i}\left(z^{k}\right)>0, H_{i}\left(z^{k}\right)>0, G_{i}\left(z^{k}\right) H_{i}\left(z^{k}\right)=\epsilon_{k}^{2}
$$

for each $k$ and each $i$. We try to estimate the index sets $\alpha\left(z^{*}\right), \beta\left(z^{*}\right)$ and $\gamma\left(z^{*}\right)$ by some index sets $\alpha^{k}, \beta^{k}$ and $\gamma^{k}$, respectively, which are obtained from $z^{k}$ and satisfy $\alpha^{k} \cup \beta^{k} \cup \gamma^{k}=\{1, \cdots, m\}$ with $\alpha^{k}, \beta^{k}, \gamma^{k}$ being mutually disjoint. Given the index sets $\alpha^{k}, \beta^{k}$ and $\gamma^{k}$, we then solve the nonlinear programming problem

$$
\begin{array}{cl}
\min & f(z) \\
\text { s.t. } & G_{i}(z) \geq 0, H_{i}(z)=0, \quad i \in \alpha^{k}, \\
& G_{i}(z)=0, H_{i}(z)=0, \quad i \in \beta^{k}, \\
& G_{i}(z)=0, H_{i}(z) \geq 0, \quad i \in \gamma^{k} \\
& g(z) \leq 0, h(z)=0 .
\end{array}
$$

This problem is no longer an MPCC and hence easier to deal with. Denote by $\hat{z}^{k}$ a stationary point of problem (3.6). Obviously, we always have $\beta^{k} \subseteq \beta\left(\hat{z}^{k}\right)$, and $\beta\left(\hat{z}^{k}\right)$ may contain some $i \in$ $\alpha^{k}$ with $G_{i}\left(\hat{z}^{k}\right)=0$ or some $i \in \gamma^{k}$ with $H_{i}\left(\hat{z}^{k}\right)=0$. If the Lagrange multipliers corresponding to the constraints

$$
\begin{array}{ll}
G_{i}(z) \geq 0, H_{i}(z)=0, & i \in \alpha^{k} \cap \beta\left(\hat{z}^{k}\right), \\
G_{i}(z)=0, H_{i}(z)=0, & i \in \beta^{k}, \\
G_{i}(z)=0, H_{i}(z) \geq 0, & i \in \gamma^{k} \cap \beta\left(\hat{z}^{k}\right)
\end{array}
$$

are all nonnegative, then $\hat{z}^{k}$ is a B-stationary point of problem (P) under the MPCC-LICQ assumption at the point [11]. Therefore, assuming that (3.6) can be solved exactly, we may terminate the method in finite steps, unlike the method in [10], which needs to solve an infinite sequence of nonlinear programs.

The key to success is to define the index sets $\alpha^{k}, \beta^{k}$ and $\gamma^{k}$ such that

$$
\alpha^{k}=\alpha\left(z^{*}\right), \beta^{k}=\beta\left(z^{*}\right), \gamma^{k}=\gamma\left(z^{*}\right)
$$

for all $k$ large enough. To this end, we may use an identification function $\rho: \Re^{n} \rightarrow[0,+\infty)$ satisfying

$$
\lim _{k \rightarrow \infty} \rho\left(z^{k}\right)=0
$$


and, for all $k$ large enough,

$$
\begin{array}{r}
\max _{i \in \beta\left(z^{*}\right)}\left\{G_{i}\left(z^{k}\right), H_{i}\left(z^{k}\right)\right\} \leq \rho\left(z^{k}\right), \\
\max _{i \in \alpha\left(z^{*}\right) \cup \gamma\left(z^{*}\right)}\left\{\min \left\{G_{i}\left(z^{k}\right), H_{i}\left(z^{k}\right)\right\}\right\} \leq \rho\left(z^{k}\right),
\end{array}
$$

and consider the following hybrid algorithm that combines the smoothing continuation method with an active set identification technique.

\section{Algorithm H:}

Step 0: Choose $\epsilon_{0}>0$ and set $k:=0$.

Step 1: Solve problem $\left(P_{\epsilon_{k}}\right)$ and denote by $z^{k}$ one of its stationary points. Set

$$
\begin{aligned}
\alpha^{k} & :=\left\{i \mid G_{i}\left(z^{k}\right)>\rho\left(z^{k}\right), \quad H_{i}\left(z^{k}\right) \leq \rho\left(z^{k}\right)\right\}, \\
\beta^{k} & :=\left\{i \mid G_{i}\left(z^{k}\right) \leq \rho\left(z^{k}\right), \quad H_{i}\left(z^{k}\right) \leq \rho\left(z^{k}\right)\right\}, \\
\gamma^{k}:=\left\{i \mid G_{i}\left(z^{k}\right) \leq \rho\left(z^{k}\right),\right. & \left.H_{i}\left(z^{k}\right)>\rho\left(z^{k}\right)\right\} .
\end{aligned}
$$

If $\alpha^{k} \cup \beta^{k} \cup \gamma^{k}=\{1, \cdots, m\}$, go to Step 2. Otherwise, go to Step 4 .

Step 2: Solve problem (3.6) to get a stationary point $\hat{z}^{k}$ and go to Step 3.

Step 3: If the Lagrange multipliers corresponding to the constraints (3.7) are all nonnegative, then terminate. Else, go to Step 4.

Step 4: Choose an $\epsilon_{k+1} \in\left(0, \epsilon_{k}\right)$ and let $k:=k+1$. Go to Step 1 .

Next, we make some remarks on the identification function $\rho$ and Algorithm $\mathrm{H}$.

First of all, we have that, if $\beta\left(z^{*}\right) \neq \emptyset$,

$$
\max _{i \in \alpha\left(z^{*}\right) \cup \gamma\left(z^{*}\right)}\left\{\min \left\{G_{i}\left(z^{k}\right), H_{i}\left(z^{k}\right)\right\}\right\}<\min _{i \in \beta\left(z^{*}\right)}\left\{G_{i}\left(z^{k}\right), H_{i}\left(z^{k}\right)\right\}
$$

holds for all $k$ large enough as long as $\alpha\left(z^{*}\right) \cup \gamma\left(z^{*}\right) \neq \emptyset$. In fact, we have from (3.5) that

$$
\begin{aligned}
\lim _{k \rightarrow \infty} \frac{\epsilon_{k}^{2}}{\min \left\{G_{i}\left(z^{k}\right), H_{i}\left(z^{k}\right)\right\}} & =\lim _{k \rightarrow \infty} \max \left\{G_{i}\left(z^{k}\right), H_{i}\left(z^{k}\right)\right\} \\
& =0, \quad i \in \beta\left(z^{*}\right)
\end{aligned}
$$

and

$$
\begin{aligned}
\lim _{k \rightarrow \infty} \frac{\epsilon_{k}^{2}}{\min \left\{G_{j}\left(z^{k}\right), H_{j}\left(z^{k}\right)\right\}} & =\lim _{k \rightarrow \infty} \max \left\{G_{j}\left(z^{k}\right), H_{j}\left(z^{k}\right)\right\} \\
& =G_{j}\left(z^{*}\right)+H_{j}\left(z^{*}\right)>0, \quad j \in \alpha\left(z^{*}\right) \cup \gamma\left(z^{*}\right) .
\end{aligned}
$$

In consequence,

$$
\lim _{k \rightarrow \infty} \frac{\min \left\{G_{j}\left(z^{k}\right), H_{j}\left(z^{k}\right)\right\}}{\min \left\{G_{i}\left(z^{k}\right), H_{i}\left(z^{k}\right)\right\}}=0
$$


holds for each $i \in \beta\left(z^{*}\right)$ and each $j \in \alpha\left(z^{*}\right) \cup \gamma\left(z^{*}\right)$ and hence we have (3.15). This inequality means that condition (3.10) implies condition (3.11) for all $k$ sufficiently large if $\beta\left(z^{*}\right) \neq \emptyset$ and $\alpha\left(z^{*}\right) \cup \gamma\left(z^{*}\right) \neq \emptyset$.

Moreover, it is obvious that $\left\{\alpha^{k}, \beta^{k}, \gamma^{k}\right\}$ defined by (3.12)-(3.14) is mutually disjoint for each $k$. On the other hand, conditions (3.10) and (3.11) ensure that, when $k$ is sufficiently large,

$$
\min \left\{G_{i}\left(z^{k}\right), H_{i}\left(z^{k}\right)\right\} \leq \rho\left(z^{k}\right), \quad \forall i .
$$

This means $\alpha^{k} \cup \beta^{k} \cup \gamma^{k}=\{1, \cdots, m\}$ and so $\left\{\alpha^{k}, \beta^{k}, \gamma^{k}\right\}$ defined in Step 1 is a partition of $\{1, \cdots, m\}$ for all $k$ sufficiently large.

Furthermore, we have from (3.9) that, when $k$ is sufficiently large,

$$
\alpha^{k} \supseteq \alpha\left(z^{*}\right), \beta^{k} \subseteq \beta\left(z^{*}\right), \gamma^{k} \supseteq \gamma\left(z^{*}\right) .
$$

In addition, it follows from (3.10) and (3.13) that $\beta\left(z^{*}\right) \subseteq \beta^{k}$ for all $k$ sufficiently large. Note that both $\left\{\alpha^{k}, \beta^{k}, \gamma^{k}\right\}$ and $\left\{\alpha\left(z^{*}\right), \beta\left(z^{*}\right), \gamma\left(z^{*}\right)\right\}$ are partitions of $\{1, \cdots, m\}$. Therefore, (3.8) holds when $k$ is large enough.

The above analysis indicates that Algorithm H may possess a finite termination property. The key question is, of course, how to define the identification function $\rho$. This is not a trivial task because the function may depend on the unknown point $z^{*}$ generally. Next, we consider the case where $\left\{z^{k}\right\}$ is asymptotically weakly nondegenerate.

Theorem 3.3 Suppose the sequence $\left\{z^{k}\right\}$ generated by Algorithm $H$ is asymptotically weakly nondegenerate. Let

$$
\rho_{1}(z):=\tau\|\min (G(z), H(z))\|^{\sigma},
$$

where $\tau>0$ and $\sigma \in(0,1)$ are constants and

$$
\min (G(z), H(z)):=\left(\min \left\{G_{1}(z), H_{1}(z)\right\}, \cdots, \min \left\{G_{m}(z), H_{m}(z)\right\}\right)^{T} .
$$

Then (3.9)-(3.11) hold with $\rho=\rho_{1}$, i.e., the function $\rho_{1}$ can serve as an identification function.

Proof: Note that the complementarity constraints (3.4) are equivalent to $\rho_{1}(z)=0$. By the fact that $\left\{z^{k}\right\}$ is convergent to $z^{*} \in \mathcal{F}$ and the continuity of $\rho_{1}$, we have

$$
\lim _{k \rightarrow \infty} \rho_{1}\left(z^{k}\right)=\rho_{1}\left(z^{*}\right)=0 .
$$

Therefore, condition (3.9) holds with $\rho=\rho_{1}$. On the other hand, for any $i \in \alpha\left(z^{*}\right) \cup \gamma\left(z^{*}\right)$, we have

$$
\min \left\{G_{i}\left(z^{k}\right), H_{i}\left(z^{k}\right)\right\} \leq\left\|\min \left(G\left(z^{k}\right), H\left(z^{k}\right)\right)\right\| \leq \rho_{1}\left(z^{k}\right)
$$

for all $k$ sufficiently large, where the first inequality follows from (3.18) and the second inequality follows from the fact that $\left\{\left\|\min \left(G\left(z^{k}\right), H\left(z^{k}\right)\right)\right\|\right\}$ is convergent to 0 and the constant $\sigma$ lies in the interval $(0,1)$. This means that $(3.11)$ with $\rho=\rho_{1}$ holds when $k$ is sufficiently large.

We next prove (3.10) with $\rho=\rho_{1}$ holds when $k$ is sufficiently large. We may assume $\beta\left(z^{*}\right) \neq \emptyset$, because (3.10) holds vacuously if $\beta\left(z^{*}\right)$ is empty. Let $i \in \beta\left(z^{*}\right)$. Since the set of accumulation points of the sequence $\left\{\nabla \Phi_{\epsilon_{k}, i}\left(z^{k}\right)\right\}$ is compact, by the asymptotically weak nondegeneracy of $\left\{z^{k}\right\}$, the set of the coefficient pairs in (3.2) is a compact subset of

$$
\Re_{++}^{2}:=\left\{(\xi, \eta)^{T} \in \Re^{2} \mid \xi>0, \eta>0\right\} .
$$


Then, by (3.1) and (3.2), there exist positive constants $a_{i}<b_{i}$ such that

$$
a_{i} \leq \frac{G_{i}\left(z^{k}\right)}{H_{i}\left(z^{k}\right)} \leq b_{i}, \quad \forall k .
$$

Let $a:=\min _{i \in \beta\left(z^{*}\right)} a_{i}$ and $b:=\max _{i \in \beta\left(z^{*}\right)} b_{i}$. It follows that

$$
0<a \leq \frac{G_{i}\left(z^{k}\right)}{H_{i}\left(z^{k}\right)} \leq b
$$

for each $k$ and each $i \in \beta\left(z^{*}\right)$. We then have from (3.19) that

$$
G_{i}\left(z^{k}\right) \leq b H_{i}\left(z^{k}\right), \quad H_{i}\left(z^{k}\right) \leq a^{-1} G_{i}\left(z^{k}\right)
$$

and so

$$
\max \left\{G_{i}\left(z^{k}\right), H_{i}\left(z^{k}\right)\right\} \leq\left(a^{-1}+b\right) \min \left\{G_{i}\left(z^{k}\right), H_{i}\left(z^{k}\right)\right\}
$$

for each $k$ and each $i \in \beta\left(z^{*}\right)$. By (3.20) and (3.18), we have

$$
\begin{aligned}
\max \left\{G_{i}\left(z^{k}\right), H_{i}\left(z^{k}\right)\right\} & \leq\left(a^{-1}+b\right)\left\|\min \left(G\left(z^{k}\right), H\left(z^{k}\right)\right)\right\| \\
& \leq \rho_{1}\left(z^{k}\right)
\end{aligned}
$$

for all $k$ sufficiently large, where the last inequality follows from the same facts as above. This completes the proof of (3.10) and so the function $\rho_{1}$ given by (3.17) can serve as an identification function.

Theorem 3.4 Suppose that the sequence $\left\{z^{k}\right\}$ generated by Algorithm $H$ is asymptotically weakly nondegenerate. Then the function

$$
\rho_{2}(z):=\tau\left\|\Phi_{0}(z)\right\|^{\sigma}, \quad \tau>0, \sigma \in(0,1)
$$

is an identification function.

Proof: Noting that, for each $i$ and each $k$,

$$
\begin{aligned}
\frac{2}{2+\sqrt{2}}\left\|\min \left(G\left(z^{k}\right), H\left(z^{k}\right)\right)\right\| & \leq\left\|\Phi_{0}\left(z^{k}\right)\right\| \\
& \leq(2+\sqrt{2})\left\|\min \left(G\left(z^{k}\right), H\left(z^{k}\right)\right)\right\|
\end{aligned}
$$

(see [24]), we have

$$
\begin{aligned}
\tau\left(\frac{2}{2+\sqrt{2}}\right)^{\sigma}\left\|\min \left(G\left(z^{k}\right), H\left(z^{k}\right)\right)\right\|^{\sigma} & \leq \rho_{2}\left(z^{k}\right) \\
& \leq \tau(2+\sqrt{2})^{\sigma}\left\|\min \left(G\left(z^{k}\right), H\left(z^{k}\right)\right)\right\|^{\sigma} .
\end{aligned}
$$

Let

$$
\begin{aligned}
& \hat{\rho}_{1}(z):=\tau\left(\frac{2}{2+\sqrt{2}}\right)^{\sigma}\|\min (G(z), H(z))\|^{\sigma}, \\
& \bar{\rho}_{1}(z):=\tau(2+\sqrt{2})^{\sigma}\|\min (G(z), H(z))\|^{\sigma} .
\end{aligned}
$$


By Theorem 3.3, both $\hat{\rho}_{1}$ and $\bar{\rho}_{1}$ are identification functions for Algorithm H. As a result, condition (3.9) holds with $\bar{\rho}_{1}$. This, together with the second inequality in (3.22), implies that condition (3.9) holds with $\rho=\rho_{2}$. On the other hand, the first inequality in (3.22), together with the fact that conditions (3.10) and (3.11) with $\rho=\hat{\rho}_{1}$ hold for all $k$ sufficiently large, means that (3.10) and (3.11) hold with $\rho=\rho_{2}$ when $k$ is sufficiently large. In consequence, the function $\rho_{2}$ satisfies conditions (3.9)-(3.11). This completes the proof.

The next lemma indicates that problem $\left(P_{\epsilon}\right)$ satisfies the standard LICQ under some appropriate assumptions, unlike problem $(\mathrm{P})$, which fails to satisfy any constraint qualification at any feasible point. A proof of the lemma may be found in [10].

Lemma 3.1 If the MPCC-LICQ holds at $z^{*}$, then there exist a neighborhood $U\left(z^{*}\right)$ of $z^{*}$ and a positive constant $\epsilon^{*}$ such that, for any $\epsilon \in\left(0, \epsilon^{*}\right)$, problem $\left(P_{\epsilon}\right)$ satisfies the standard LICQ at any feasible point in $U\left(z^{*}\right)$.

Summarizing the above arguments, we obtain the following concluding result.

Theorem 3.5 Let $\sigma \in(0,1), \tau>0$, and $\rho$ be $\rho_{1}$ defined by (3.17) or $\rho_{2}$ defined by (3.21). Suppose that the sequence $\left\{z^{k}\right\}$ of stationary points of problems $\left(P_{\epsilon_{k}}\right)$ converges to $z^{*}$ as $\epsilon_{k} \rightarrow 0$, the MPCC-LICQ holds at $z^{*}$, and the sequence $\left\{z^{k}\right\}$ is asymptotically weakly nondegenerate. Then, for all $k$ sufficiently large, we have

(i) problem $\left(P_{\epsilon_{k}}\right)$ satisfies the standard LICQ at $z^{k}$;

(ii) the sets $\alpha^{k}, \beta^{k}$, and $\gamma^{k}$ defined by (3.12), (3.13), and (3.14) satisfy (3.8).

This theorem indicates that, by means of the technique introduced above, we can identify the active sets $\alpha\left(z^{*}\right), \beta\left(z^{*}\right)$, and $\gamma\left(z^{*}\right)$ in a finite number of iterations under some mild conditions. As a result, if the problem

$$
\begin{array}{cl}
\min & f(z) \\
\text { s.t. } & G_{i}(z) \geq 0, H_{i}(z)=0, \quad i \in \alpha\left(z^{*}\right), \\
& G_{i}(z)=0, H_{i}(z)=0, \quad i \in \beta\left(z^{*}\right), \\
& G_{i}(z)=0, H_{i}(z) \geq 0, \quad i \in \gamma\left(z^{*}\right), \\
& g(z) \leq 0, h(z)=0
\end{array}
$$

can be solved exactly, we may expect that Algorithm H terminates in finite steps. In particular, if $z^{*}$ is a stationary point obtained by solving (3.23), then, under the assumptions of either Theorem 3.1 or Theorem 3.2, the algorithm terminates in a finite number of iterations by producing a B-stationary point of problem $(\mathrm{P})$. Note that it is possible that the algorithm terminates by producing a B-stationary point of $(\mathrm{P})$ before we solve problem (3.23).

Remark 3.1 In order to ensure $\alpha^{k} \cup \beta^{k} \cup \gamma^{k}=\{1, \cdots, m\}$ in Step 1 for each $k$, we may define

$$
\beta^{k}:=\{1, \cdots, m\} \backslash\left(\alpha^{k} \cup \gamma^{k}\right)
$$

instead of (3.13) in Algorithm H. 
Remark 3.2 Under the assumption of asymptotically weak nondegeneracy of $\left\{z^{k}\right\}$, we may define $\left\{\alpha^{k}, \beta^{k}, \gamma^{k}\right\}$ in a different manner in Step 1. For example, we may put

$$
\begin{aligned}
& \alpha^{k}:=\left\{i \mid G_{i}\left(z^{k}\right)>\sqrt{\epsilon_{k}}, \quad H_{i}\left(z^{k}\right) \leq \sqrt{\epsilon_{k}}\right\}, \\
& \beta^{k}:=\left\{i \mid G_{i}\left(z^{k}\right) \leq \sqrt{\epsilon_{k}}, \quad H_{i}\left(z^{k}\right) \leq \sqrt{\epsilon_{k}}\right\}, \\
& \gamma^{k}:=\left\{i \mid G_{i}\left(z^{k}\right) \leq \sqrt{\epsilon_{k}}, \quad H_{i}\left(z^{k}\right)>\sqrt{\epsilon_{k}}\right\}
\end{aligned}
$$

instead of (3.12)-(3.14). In fact, the proof of Theorem 3.3 indicates that there exist two positive numbers $a$ and $b$ such that (3.19) holds for each $i \in \beta\left(z^{*}\right)$ and each $k$. Therefore, by the equality in (3.5) and the asymptotically weak nondegeneracy, we deduce

$$
\begin{aligned}
& G_{i}\left(z^{k}\right)=O\left(\epsilon_{k}\right), \quad H_{i}\left(z^{k}\right)=O\left(\epsilon_{k}\right), \quad i \in \beta\left(z^{*}\right), \\
& \min \left\{G_{i}\left(z^{k}\right), H_{i}\left(z^{k}\right)\right\}=O\left(\epsilon_{k}^{2}\right), \quad i \in \alpha\left(z^{*}\right) \cup \gamma\left(z^{*}\right) .
\end{aligned}
$$

Since $\left\{\sqrt{\epsilon_{k}}\right\}$ approaches zero slower than both $\left\{\epsilon_{k}\right\}$ and $\left\{\epsilon_{k}^{2}\right\}$, we have from (3.27)-(3.28) that

$$
\min \left\{G_{i}\left(z^{k}\right), H_{i}\left(z^{k}\right)\right\} \leq \sqrt{\epsilon_{k}}
$$

for each $i$ and each $k$ sufficiently large. This means $\alpha^{k} \cup \beta^{k} \cup \gamma^{k}=\{1, \cdots, m\}$ when $k$ is sufficiently large. Noticing that $\left\{\alpha^{k}, \beta^{k}, \gamma^{k}\right\}$ defined by (3.24)-(3.26) is mutually disjoint for each $k$, we have that $\left\{\alpha^{k}, \beta^{k}, \gamma^{k}\right\}$ given by (3.24)-(3.26) is a partition of $\{1, \cdots, m\}$ for all $k$ sufficiently large. On the other hand, it is obvious that, when $k$ is sufficiently large,

$$
\alpha^{k} \supseteq \alpha\left(z^{*}\right), \beta^{k} \subseteq \beta\left(z^{*}\right), \gamma^{k} \supseteq \gamma\left(z^{*}\right) .
$$

Moreover, it follows from (3.25) and (3.27) that $\beta\left(z^{*}\right) \subseteq \beta^{k}$ for all $k$ sufficiently large. Note that both $\left\{\alpha^{k}, \beta^{k}, \gamma^{k}\right\}$ and $\left\{\alpha\left(z^{*}\right), \beta\left(z^{*}\right), \gamma\left(z^{*}\right)\right\}$ are partitions of $\{1, \cdots, m\}$. Therefore, (3.8) holds when $k$ is large enough. So, we may employ (3.24)-(3.26) instead of (3.12)-(3.14) in Step 1 of Algorithm H. From the computational point of view, (3.24)-(3.26) are simpler than (3.12)-(3.14).

\subsection{Modified hybrid method with index addition strategy}

For Algorithm $\mathrm{H}$, the asymptotically weak nondegeneracy condition is a key assumption. Although this condition is not excessively stringent because it is implied by the ULSC condition, it is certainly desirable to lessen the required assumptions. In this subsection, we introduce a modified method with index addition strategy and in the next subsection, we describe another method with the converse strategy. Both of these two methods do not require the assumption of asymptotically weak nondegeneracy.

Let $\rho$ be a function defined by (3.17) or (3.21) with $\tau>0$ and $\sigma \in(0,1)$.

\section{Algorithm HIA:}

Step 0: Choose $\theta_{0}>0$ and $\epsilon_{0}>0$. Set $k:=0$. 
Step 1: Solve problem $\left(P_{\epsilon_{k}}\right)$ to obtain a stationary point $z^{k}$ and set

$$
\begin{aligned}
& \alpha_{0}^{k}:=\left\{i \mid \quad G_{i}\left(z^{k}\right)>\rho\left(z^{k}\right), \quad H_{i}\left(z^{k}\right) \leq \rho\left(z^{k}\right)\right\}, \\
& \beta_{0}^{k}:=\left\{i \mid \quad G_{i}\left(z^{k}\right) \leq \rho\left(z^{k}\right), \quad H_{i}\left(z^{k}\right) \leq \rho\left(z^{k}\right)\right\}, \\
& \gamma_{0}^{k}:=\{1, \cdots, m\} \backslash\left(\alpha_{0}^{k} \cup \beta_{0}^{k}\right), \\
& \delta_{0}^{k}:=+\infty,
\end{aligned}
$$

and $j:=0$. Go to Step 2 .

Step 2: Solve the problem

$$
\begin{array}{cl}
\min & f(z) \\
\text { s.t. } & G_{i}(z) \geq 0, H_{i}(z)=0, \quad i \in \alpha_{j}^{k}, \\
& G_{i}(z)=0, H_{i}(z)=0, \quad i \in \beta_{j}^{k}, \\
& G_{i}(z)=0, H_{i}(z) \geq 0, \quad i \in \gamma_{j}^{k}, \\
& g(z) \leq 0, h(z)=0
\end{array}
$$

to get a stationary point $\hat{z}_{j}^{k}$.

Step 3: If, in (3.32), the Lagrange multipliers corresponding to the constraints

$$
\begin{aligned}
& G_{i}(z) \geq 0, H_{i}(z)=0, \quad i \in \alpha_{j}^{k} \cap \beta\left(\hat{z}_{j}^{k}\right), \\
& G_{i}(z)=0, H_{i}(z)=0, \quad i \in \beta_{j}^{k}, \\
& G_{i}(z)=0, H_{i}(z) \geq 0, \quad i \in \gamma_{j}^{k} \cap \beta\left(\hat{z}_{j}^{k}\right)
\end{aligned}
$$

are all nonnegative, then terminate. Else, if there is an $\hat{i} \in \alpha_{j}^{k} \cup \gamma_{j}^{k}$ such that

$$
\min _{i \in \alpha_{j}^{k} \cup \gamma_{j}^{k}}\left\{\max \left\{G_{i}\left(z^{k}\right), H_{i}\left(z^{k}\right)\right\}\right\}=\max \left\{G_{\hat{i}}\left(z^{k}\right), H_{\hat{i}}\left(z^{k}\right)\right\}<\theta_{k},
$$

then set

$$
\delta_{j+1}^{k}:=\min \left\{\delta_{j}^{k}, \frac{1}{2} \min \left\{G_{i}\left(\hat{z}_{j}^{k}\right)+H_{i}\left(\hat{z}_{j}^{k}\right) \mid i \in \alpha\left(\hat{z}_{j}^{k}\right) \cup \gamma\left(\hat{z}_{j}^{k}\right)\right\}\right\}
$$

and

$$
\begin{aligned}
\alpha_{j+1}^{k} & :=\alpha_{j}^{k} \backslash\{\hat{i}\}, \\
\beta_{j+1}^{k} & :=\beta_{j}^{k} \cup\{\hat{i}\}, \\
\gamma_{j+1}^{k} & :=\gamma_{j}^{k} \backslash\{\hat{i}\}, \\
j & :=j+1
\end{aligned}
$$

and go to Step 2. Otherwise, let $j_{k}:=j$ and $\delta_{k}:=\delta_{j}^{k}$ and go to Step 4 .

Step 4: Choose $\epsilon_{k+1} \in\left(0, \epsilon_{k}\right)$ and set $\theta_{k+1}:=\min \left\{\theta_{k}, \delta_{k}\right\}$. Go to Step 1 with $k:=k+1$. 
The next theorem describes the relations between the sets $\left\{\alpha_{j}^{k}, \beta_{j}^{k}, \gamma_{j}^{k}\right\}, j=0,1, \cdots, j_{k}$, and $\left\{\alpha\left(z^{*}\right), \beta\left(z^{*}\right), \gamma\left(z^{*}\right)\right\}$.

Theorem 3.6 Suppose that the sequence $\left\{z^{k}\right\}$ generated by Algorithm HIA converges to $z^{*}$ as $\epsilon_{k} \rightarrow 0$. Then there is an integer $k_{0} \geq 0$ such that, for any $k \geq k_{0}$,

(i) $\alpha_{0}^{k} \supseteq \alpha\left(z^{*}\right), \beta_{0}^{k} \subseteq \beta\left(z^{*}\right), \gamma_{0}^{k} \supseteq \gamma\left(z^{*}\right)$;

(ii) if $\beta\left(z^{*}\right)=\emptyset$, namely, $z^{*}$ is nondegenerate, then we have

$$
\alpha_{0}^{k}=\alpha\left(z^{*}\right), \quad \beta_{0}^{k}=\emptyset, \quad \gamma_{0}^{k}=\gamma\left(z^{*}\right) ;
$$

(iii) if $\beta\left(z^{*}\right) \neq \emptyset$ and, for some $j, \beta_{j}^{k}$ is a proper subset of $\beta\left(z^{*}\right)$, then any index $\hat{i} \in \alpha_{j}^{k} \cup \gamma_{j}^{k}$ satisfying (3.34) belongs to $\beta\left(z^{*}\right)$ and hence we have

$$
\begin{aligned}
& \alpha_{0}^{k} \supseteq \alpha_{1}^{k} \supseteq \cdots \supseteq \alpha_{j}^{k} \supseteq \alpha_{j+1}^{k} \supseteq \alpha\left(z^{*}\right), \\
& \beta_{0}^{k} \subseteq \beta_{1}^{k} \subseteq \cdots \subseteq \beta_{j}^{k} \subseteq \beta_{j+1}^{k} \subseteq \beta\left(z^{*}\right), \\
& \gamma_{0}^{k} \supseteq \gamma_{1}^{k} \supseteq \cdots \supseteq \gamma_{j}^{k} \supseteq \gamma_{j+1}^{k} \supseteq \gamma\left(z^{*}\right) .
\end{aligned}
$$

Proof: First of all, we note that

$$
\begin{aligned}
& \alpha_{0}^{k} \supseteq \alpha_{1}^{k} \supseteq \cdots \supseteq \alpha_{j}^{k} \supseteq \alpha_{j+1}^{k}, \\
& \beta_{0}^{k} \subseteq \beta_{1}^{k} \subseteq \cdots \subseteq \beta_{j}^{k} \subseteq \beta_{j+1}^{k}, \\
& \gamma_{0}^{k} \supseteq \gamma_{1}^{k} \supseteq \cdots \supseteq \gamma_{j}^{k} \supseteq \gamma_{j+1}^{k}
\end{aligned}
$$

by (3.36)-(3.38). Next we show the existence of the integer $k_{0}$. We only consider the case where $\rho=\rho_{2}$. We may deal with the other case similarly.

Since $\left\{z^{k}\right\}$ converges to $z^{*} \in \mathcal{F}$, we have from the continuity of the function $\rho$ that

$$
\lim _{k \rightarrow \infty} \rho\left(z^{k}\right)=0
$$

and hence, for each $i \in \alpha\left(z^{*}\right) \cup \gamma\left(z^{*}\right)$,

$$
\begin{aligned}
\max \left\{G_{i}\left(z^{k}\right), H_{i}\left(z^{k}\right)\right\} & >\rho\left(z^{k}\right) \quad\left(:=\tau\left\|\Phi_{0}\left(z^{k}\right)\right\|^{\sigma}\right) \\
& \geq 2\left\|\Phi_{0}\left(z^{k}\right)\right\| \\
& \geq 2 \phi_{0}\left(G_{i}\left(z^{k}\right), H_{i}\left(z^{k}\right)\right) \\
& =2\left(G_{i}\left(z^{k}\right)+H_{i}\left(z^{k}\right)-\sqrt{\left(G_{i}\left(z^{k}\right)\right)^{2}+\left(H_{i}\left(z^{k}\right)\right)^{2}}\right) \\
& =\frac{4 G_{i}\left(z^{k}\right) H_{i}\left(z^{k}\right)}{G_{i}\left(z^{k}\right)+H_{i}\left(z^{k}\right)+\sqrt{\left(G_{i}\left(z^{k}\right)\right)^{2}+\left(H_{i}\left(z^{k}\right)\right)^{2}}} \\
& \geq \min \left\{G_{i}\left(z^{k}\right), H_{i}\left(z^{k}\right)\right\}
\end{aligned}
$$

when $k$ is large enough, where the second inequality follows from the fact that $\left\{\left\|\Phi_{0}\left(z^{k}\right)\right\|\right\}$ is convergent to 0 and the constant $\sigma$ lies in the interval $(0,1)$, and the last inequality follows from (3.5) and the fact that

$$
\begin{aligned}
G_{i}\left(z^{k}\right)+H_{i}\left(z^{k}\right)+\sqrt{\left(G_{i}\left(z^{k}\right)\right)^{2}+\left(H_{i}\left(z^{k}\right)\right)^{2}} & \leq 2\left(G_{i}\left(z^{k}\right)+H_{i}\left(z^{k}\right)\right) \\
& \leq 4 \max \left\{G_{i}\left(z^{k}\right), H_{i}\left(z^{k}\right)\right\} .
\end{aligned}
$$


Thus, we have from (3.44)-(3.45) and the continuity of the functions $G$ and $H$ that, for any $k$ sufficiently large,

$$
\begin{aligned}
& G_{i}\left(z^{k}\right)>\rho\left(z^{k}\right), \quad H_{i}\left(z^{k}\right) \leq \rho\left(z^{k}\right), \quad i \in \alpha\left(z^{*}\right), \\
& G_{i}\left(z^{k}\right) \leq \rho\left(z^{k}\right), \quad H_{i}\left(z^{k}\right)>\rho\left(z^{k}\right), \quad i \in \gamma\left(z^{*}\right) .
\end{aligned}
$$

Note that (3.29)-(3.31) imply

$$
\gamma_{0}^{k} \supseteq\left\{i \mid \quad G_{i}\left(z^{k}\right) \leq \rho\left(z^{k}\right), \quad H_{i}\left(z^{k}\right)>\rho\left(z^{k}\right)\right\}
$$

for each $k$. Moreover, it is obvious from (3.43) that $\beta_{0}^{k} \subseteq \beta\left(z^{*}\right)$ when $k$ is sufficiently large. It then follows from (3.29)-(3.31) and (3.46)-(3.48) that there exists an integer $k_{1} \geq 0$ such that, for any $k \geq k_{1}$,

$$
\alpha_{0}^{k} \supseteq \alpha\left(z^{*}\right), \beta_{0}^{k} \subseteq \beta\left(z^{*}\right), \gamma_{0}^{k} \supseteq \gamma\left(z^{*}\right) .
$$

If $z^{*}$ is nondegenerate, then (3.39) follows from (3.49) and the fact that both $\left\{\alpha_{0}^{k}, \beta_{0}^{k}, \gamma_{0}^{k}\right\}$ and $\left\{\alpha\left(z^{*}\right), \beta\left(z^{*}\right), \gamma\left(z^{*}\right)\right\}$ are partitions of the set $\{1, \cdots, m\}$. Therefore, (i) and (ii) hold for all $k \geq k_{1}$.

Suppose $\beta\left(z^{*}\right) \neq \emptyset$. If $\beta\left(z^{*}\right)=\{1, \cdots, m\}$, then we have $\beta_{j+1}^{k} \subseteq \beta\left(z^{*}\right)$ for any $k$. Next we suppose that $\beta\left(z^{*}\right)$ is a proper subset of $\{1, \cdots, m\}$. We will show that, when $k$ is large enough, if there is an $\hat{i} \in \alpha_{j}^{k} \cup \gamma_{j}^{k}$ satisfying (3.34) and $\beta_{j}^{k}$ is a proper subset of $\beta\left(z^{*}\right)$, then $\hat{i} \in \beta\left(z^{*}\right)$. Note that

$$
\begin{aligned}
\lim _{k \rightarrow \infty} \max \left\{G_{i}\left(z^{k}\right), H_{i}\left(z^{k}\right)\right\}=G_{i}\left(z^{*}\right)+H_{i}\left(z^{*}\right)>0, & i \notin \beta\left(z^{*}\right), \\
\lim _{k \rightarrow \infty} \max \left\{G_{i}\left(z^{k}\right), H_{i}\left(z^{k}\right)\right\}=0, & i \in \beta\left(z^{*}\right) .
\end{aligned}
$$

We have from (3.50) and (3.51) that there exists an integer $k_{0} \geq k_{1}$ such that, for any $k \geq k_{0}$,

$$
\max _{i \in \beta\left(z^{*}\right)}\left\{\max \left\{G_{i}\left(z^{k}\right), H_{i}\left(z^{k}\right)\right\}\right\}<\min _{i \notin \beta\left(z^{*}\right)}\left\{\max \left\{G_{i}\left(z^{k}\right), H_{i}\left(z^{k}\right)\right\}\right\} .
$$

This inequality means that the index $\hat{i}$ satisfying (3.34) must be in $\beta\left(z^{*}\right)$ as long as $\beta_{j}^{k}$ is a proper subset of $\beta\left(z^{*}\right)$, namely,

$$
\left(\alpha_{j}^{k} \cup \gamma_{j}^{k}\right) \cap \beta\left(z^{*}\right)=\beta\left(z^{*}\right) \backslash \beta_{j}^{k} \neq \emptyset .
$$

By (3.37), we have $\beta_{j+1}^{k} \subseteq \beta\left(z^{*}\right)$ and therefore, (3.40)-(3.42) hold for all $k \geq k_{0}$. Note that, since $k_{0} \geq k_{1}$, (i) and (ii) also hold for all $k \geq k_{0}$. This completes the proof.

We proceed to analyzing convergence properties of Algorithm HIA in detail. First, we make the following assumption:

A1: Even if the identical subproblem appears in Step 2 at infinitely many iterations and this problem may have an infinite number of solutions, we always obtain the same solution, or at most finitely many different solutions. 
This assumption seems reasonable in practice, since an iterative method applied to solve a subproblem will generate an identical sequence as long as the same starting point is chosen. of

In Algorithm HIA, for each $k$, the parameter $\theta_{k}$ is expectantly used as a positive lower bound

$$
\min _{i \notin \beta\left(z^{*}\right)} \max \left\{G_{i}\left(z^{*}\right), H_{i}\left(z^{*}\right)\right\}=\min _{i \notin \beta\left(z^{*}\right)}\left(G_{i}\left(z^{*}\right)+H_{i}\left(z^{*}\right)\right)>0 .
$$

In fact, a key technique for obtaining a finite termination property of Algorithm HIA is to choose the index sets $\alpha_{j}^{k}, \beta_{j}^{k}$ and $\gamma_{j}^{k}$ so that

$$
\alpha_{j_{k}^{\prime}}^{k}=\alpha\left(z^{*}\right), \beta_{j_{k}^{\prime}}^{k}=\beta\left(z^{*}\right), \gamma_{j_{k}^{\prime}}^{k}=\gamma\left(z^{*}\right)
$$

hold for some index $j_{k}^{\prime} \in\left\{0,1, \cdots, j_{k}\right\}$ when $k$ is large enough. In order to ensure this, the number $\theta_{k}$ needs to be small enough to exclude all the indices in $\alpha\left(z^{*}\right) \cup \gamma\left(z^{*}\right)$ from $\beta_{j_{k}^{\prime}}^{k}$ for all $k$ sufficiently large. Another requirement is that all the indices in $\beta\left(z^{*}\right)$ remain in $\beta_{j_{k}^{\prime}}^{k}$ when $k$ is sufficiently large.

Since the index set $\{1, \cdots, m\}$ has a finite number of partitions, there are a finite number of subproblems (3.32). By Assumption A1, the set

$$
S:=\left\{\hat{z}_{j}^{k} \mid \quad 0 \leq j \leq j_{k}, k=0,1, \cdots\right\}
$$

is a finite set. Recall that $\hat{z}_{j}^{k} \in \mathcal{F}$ for any $k$ and $j$. We consider the following two cases.

Case I: $\quad \bigcup_{\hat{z}_{j}^{k} \in S}\left(\alpha\left(\hat{z}_{j}^{k}\right) \cup \gamma\left(\hat{z}_{j}^{k}\right)\right) \neq \emptyset$. In this case, we have

$$
\min _{\hat{z}_{j}^{k} \in S}\left\{G_{i}\left(\hat{z}_{j}^{k}\right)+H_{i}\left(\hat{z}_{j}^{k}\right) \mid i \in \alpha\left(\hat{z}_{j}^{k}\right) \cup \gamma\left(\hat{z}_{j}^{k}\right)\right\}>0,
$$

since $S$ is a finite set. It then follows from (3.35) and the way of updating $\delta_{k}$ in Step 3 that the parameter $\delta_{k}$ stays at a positive constant when $k$ is sufficiently large. So, by the updating rule of $\theta_{k}$, there exists an integer $\bar{k}>0$ such that

$$
\theta_{k}=\theta_{\bar{k}}>0, \quad k \geq \bar{k}
$$

Since

$$
\lim _{k \rightarrow \infty} \max \left\{G_{i}\left(z^{k}\right), H_{i}\left(z^{k}\right)\right\}=0, \quad \forall i \in \beta\left(z^{*}\right)
$$

we have

$$
\max \left\{G_{i}\left(z^{k}\right), H_{i}\left(z^{k}\right)\right\}<\theta_{\bar{k}}, \quad \forall i \in \beta\left(z^{*}\right)
$$

for all sufficiently large $k$. Moreover, by the definition of $\beta\left(z^{*}\right)$, we have

$$
\max _{i \in \beta\left(z^{*}\right)}\left\{\max \left\{G_{i}\left(z^{k}\right), H_{i}\left(z^{k}\right)\right\}\right\}<\min _{i \notin \beta\left(z^{*}\right)}\left\{\max \left\{G_{i}\left(z^{k}\right), H_{i}\left(z^{k}\right)\right\}\right\}
$$

for any $k$ large enough. Taking into account (3.34), we deduce from (3.57) and (3.58) that the indices in $\beta\left(z^{*}\right)$ are inevitably included by some $\beta_{j}^{k}$ and, in Step 3, these indices must be chosen earlier than the indices in $\alpha\left(z^{*}\right) \cup \gamma\left(z^{*}\right)$. As a result, there is some $j \in\left\{0,1, \cdots, j_{k}\right\}$ such that 
$\beta\left(z^{*}\right) \subseteq \beta_{j}^{k}$ whenever $k$ is sufficiently large. This together with Theorem 3.6 means that, when $k$ is large sufficiently, there must be some index $j_{k}^{\prime} \in\left\{0,1, \cdots, j_{k}\right\}$ satisfying (3.54), and then problem (3.32) with $j=j_{k}^{\prime}$ is actually equivalent to problem (3.23). As long as a solution of problem (3.32) with $j=j_{k}^{\prime}$ yields a B-stationary point of problem (P), Algorithm HIA may terminate in a finite number of iterations. Of course, it may happen that the algorithm stops by getting another B-stationary point of $(\mathrm{P})$ before we identify the correct index sets.

Furthermore, we make the following assumption, which is most likely to hold when problem (P) has finitely many B-stationary points:

A2: The limit point $z^{*}$ of $\left\{z^{k}\right\}$, which is a sequence of stationary points of $\left(P_{\epsilon_{k}}\right)$, is a B-stationary point of problem (P) and it belongs to the set $S$ given by (3.55).

Then, it follows from (3.35) and the updating rule of $\theta_{k}$ that the number $\theta_{\bar{k}}$ in (3.56) is actually a positive lower bound of (3.53). Thus, our requirements are fulfilled: That is, in Step 3,

(a) all the indices in $\alpha\left(z^{*}\right) \cup \gamma\left(z^{*}\right)$ are excluded from $\beta_{j_{k}}^{k}$ eventually;

(b) all the indices in $\beta\left(z^{*}\right)$ remain in $\beta_{j_{k}}^{k}$ eventually.

Therefore, we are able to identify the correct index sets $\alpha\left(z^{*}\right), \beta\left(z^{*}\right)$, and $\gamma\left(z^{*}\right)$ in a finite number of iterations and furthermore, we may terminate the algorithm finitely by getting $z^{*}$. Note that Algorithm HIA may stop prematurely by producing another B-stationary point of problem $(\mathrm{P})$.

Case II: $\quad \alpha\left(\hat{z}_{j}^{k}\right) \cup \gamma\left(\hat{z}_{j}^{k}\right)=\emptyset, \forall \hat{z}_{j}^{k} \in S$. In this case, we have from the updating rules of $\delta_{k}$ and $\theta_{k}$ that $\delta_{k}$ remains to be $+\infty$ and so

$$
\theta_{k} \equiv \theta_{0}, \quad \forall k
$$

Since the strategy in Algorithm HIA is to add some indices to $\beta_{j}^{k}$ one after another, by Theorem 3.6, we have the same conclusion as in Case I: When $k$ is sufficiently large, there exists some index $j_{k}^{\prime} \in\left\{0,1, \cdots, j_{k}\right\}$ satisfying (3.54). Furthermore, suppose that Assumption A2 holds. In the present case, this means

$$
\beta\left(z^{*}\right)=\{1,2, \cdots, m\} .
$$

Recall that $\theta_{k} \equiv \theta_{0}$ for all $k$. As a result, all indices should be chosen to be in $\beta_{j_{k}}^{k}$ eventually when $k$ becomes large enough, i.e., we also can identify the index sets $\alpha\left(z^{*}\right), \beta\left(z^{*}\right)$, and $\gamma\left(z^{*}\right)$ in a finite number of steps.

The preceding analysis together with Lemma 3.1 yields the following concluding result.

Theorem 3.7 Suppose that the sequence $\left\{z^{k}\right\}$ generated by Algorithm HIA converges to $z^{*}$ as $\epsilon_{k} \rightarrow 0$ and the MPCC-LICQ holds at $z^{*}$. Then, under Assumption A1, we have that, for any sufficiently large $k$,

(i) problem $\left(P_{\epsilon_{k}}\right)$ satisfies the standard LICQ at $z^{k}$;

(ii) there exists $j_{k}^{\prime} \in\left\{0,1, \cdots, j_{k}\right\}$ such that $\alpha_{j_{k}^{\prime}}^{k}, \beta_{j_{k}^{\prime}}^{k}$, and $\gamma_{j_{k}^{\prime}}^{k}$ satisfy condition (3.54).

If furthermore, Assumption A2 also holds, then

$$
\alpha_{j_{k}}^{k}=\alpha\left(z^{*}\right), \beta_{j_{k}}^{k}=\beta\left(z^{*}\right), \gamma_{j_{k}}^{k}=\gamma\left(z^{*}\right)
$$

hold for all $k$ sufficiently large. 
In consequence, without the assumption of asymptotically weak nondegeneracy, we have attained the same target as Algorithm $\mathrm{H}$, which is to identify the index sets $\alpha\left(z^{*}\right), \beta\left(z^{*}\right)$, and $\gamma\left(z^{*}\right)$ finitely. Thus, Algorithm HIA may terminate in a finite number of iterations by producing a B-stationary point of problem $(\mathrm{P})$.

On the other hand, if the sequence $\left\{z^{k}\right\}$ is asymptotically weakly nondegenerate, the sets $\alpha_{0}^{k}, \beta_{0}^{k}, \gamma_{0}^{k}$ given in Step 1 of Algorithm HIA are the same as the sets $\alpha^{k}, \beta^{k}, \gamma^{k}$ given in Step 1 of Algorithm $\mathrm{H}$ when $k$ is large enough. Theorem 3.5(ii) immediately yields the following result.

Theorem 3.8 Suppose the sequence $\left\{z^{k}\right\}$ generated by Algorithm HIA converges to $z^{*}$ as $\epsilon_{k} \rightarrow$ 0 , the MPCC-LICQ holds at $z^{*}$, and $\left\{z^{k}\right\}$ is asymptotically weakly nondegenerate. Then the sets $\alpha_{0}^{k}, \beta_{0}^{k}$, and $\gamma_{0}^{k}$ satisfy (3.54) with $j_{k}^{\prime}=0$ when $k$ is large enough.

The main strategy in Algorithm HIA is to add some indices, which are chosen from $\alpha_{j}^{k} \cup \gamma_{j}^{k}$, to $\beta_{j}^{k}$. Since $\alpha_{j}^{k} \cup \gamma_{j}^{k}$ contains $\beta\left(z^{*}\right)$ for any $k$ large enough, condition (3.54) holds for some $j_{k}^{\prime} \in\left\{0,1, \cdots, j_{k}\right\}$ when $k$ is large enough. In order to ensure this, the inclusions in (3.49) are necessary. In the above discussion, we suppose that $\tau>0$ and $\sigma \in(0,1)$, just as in Algorithm H. Actually, from the proof of Theorem 3.6, (3.49) remains true for the case where $\tau \geq 2$ and $\sigma=1$ and furthermore, so do Theorems 3.6 and 3.7. Moreover, since the functions $G$ and $H$ play a symmetric role in problem (P), we may exchange the definitions of $\alpha_{0}^{k}$ and $\gamma_{0}^{k}$ in Step 1 of Algorithm HIA, namely, let

$$
\begin{aligned}
\gamma_{0}^{k} & :=\left\{i \mid \quad G_{i}\left(z^{k}\right) \leq \rho\left(z^{k}\right), \quad H_{i}\left(z^{k}\right)>\rho\left(z^{k}\right)\right\}, \\
\alpha_{0}^{k} & :=\{1, \cdots, m\} \backslash\left(\beta_{0}^{k} \cup \gamma_{0}^{k}\right)
\end{aligned}
$$

instead of (3.31) and (3.29), respectively.

\subsection{Modified hybrid method with index subtraction strategy}

In this subsection, we consider another hybrid algorithm that adopts an index subtraction strategy. One advantage of this algorithm is that the function $\rho$ employed in the last subsection can be replaced by a sequence of positive numbers.

\section{Algorithm HIS:}

Step 0: Choose $\eta>0, \theta_{0}>0, \xi_{0}>0$, and $\epsilon_{0}>0$. Set $k:=0$.

Step 1: Solve problem $\left(P_{\epsilon_{k}}\right)$ and let $z^{k}$ denote one of its stationary points. Set

$$
\begin{aligned}
& \alpha_{0}^{k}:=\left\{i \mid \quad G_{i}\left(z^{k}\right)>\eta, \quad H_{i}\left(z^{k}\right) \leq \xi_{k}\right\}, \\
& \gamma_{0}^{k}:=\left\{i \mid G_{i}\left(z^{k}\right) \leq \xi_{k}, \quad H_{i}\left(z^{k}\right)>\eta\right\} \backslash \alpha_{0}^{k} \text {, } \\
& \beta_{0}^{k}:=\{1, \cdots, m\} \backslash\left(\alpha_{0}^{k} \cup \gamma_{0}^{k}\right) \text {, } \\
& \delta_{0}^{k}:=+\infty,
\end{aligned}
$$

and $j:=0$. Go to Step 2 .

Step 2: If problem (3.32) is solvable, let $\hat{z}_{j}^{k}$ denote one of its stationary points and go to Step 3. Otherwise, go to Step 4. 
Step 3: If, in (3.32), the Lagrange multipliers corresponding to the constraints (3.33) are all nonnegative, then terminate. Else, if there is an $\hat{i} \in \beta_{j}^{k}$ such that

$$
\max _{i \in \beta_{j}^{k}}\left\{\max \left\{G_{i}\left(z^{k}\right), H_{i}\left(z^{k}\right)\right\}\right\}=\max \left\{G_{\hat{i}}\left(z^{k}\right), H_{\hat{i}}\left(z^{k}\right)\right\}>\theta_{k},
$$

then set

$$
\delta_{j+1}^{k}:=\min \left\{\delta_{j}^{k}, \frac{1}{2} \min \left\{G_{i}\left(\hat{z}_{j}^{k}\right)+H_{i}\left(\hat{z}_{j}^{k}\right) \mid i \in \alpha\left(\hat{z}_{j}^{k}\right) \cup \gamma\left(\hat{z}_{j}^{k}\right)\right\}\right\}
$$

and

$$
\begin{aligned}
& \alpha_{j+1}^{k}:= \begin{cases}\alpha_{j}^{k} \cup\{\hat{i}\}, & \text { if } G_{\hat{i}}\left(z^{k}\right) \geq H_{\hat{i}}\left(z^{k}\right) \\
\alpha_{j}^{k}, & \text { otherwise, }\end{cases} \\
& \beta_{j+1}^{k}:=\beta_{j}^{k} \backslash\{\hat{i}\}, \\
& \gamma_{j+1}^{k}:= \begin{cases}\gamma_{j}^{k}, & \text { if } G_{\hat{i}}\left(z^{k}\right) \geq H_{\hat{i}}\left(z^{k}\right) \\
\gamma_{j}^{k} \cup\{\hat{i}\}, & \text { otherwise, }\end{cases} \\
& j:=j+1
\end{aligned}
$$

and go to Step 2. Otherwise, let $j_{k}:=j$ and $\delta_{k}:=\delta_{j}^{k}$ and go to Step 4 .

Step 4: Choose $\epsilon_{k+1} \in\left(0, \epsilon_{k}\right), \xi_{k+1} \in\left(0, \xi_{k}\right]$, and set $\theta_{k+1}:=\min \left\{\theta_{k}, \delta_{k}\right\}$. Let $k:=k+1$ and go to Step 1.

Note that, since $G_{i}\left(z^{k}\right) \rightarrow 0$ for each $i \in \beta\left(z^{*}\right) \cup \gamma\left(z^{*}\right)$, the index set $\alpha_{0}^{k}$ determined in Step 1 will eventually consist of indices in $\alpha\left(z^{*}\right)$ only. Similarly, $\gamma_{0}^{k}$ will eventually consist of indices in $\gamma\left(z^{*}\right)$ only. Therefore, we must have

$$
\alpha_{0}^{k} \subseteq \alpha\left(z^{*}\right), \beta_{0}^{k} \supseteq \beta\left(z^{*}\right), \gamma_{0}^{k} \subseteq \gamma\left(z^{*}\right)
$$

for all $k$ sufficiently large. This is the key requirement for Algorithm HIS, which subtracts some indices from $\beta_{j}^{k}$ so that condition (3.54) holds for some $j_{k}^{\prime} \in\left\{0,1, \cdots, j_{k}\right\}$ when $k$ is large enough.

In Algorithm HIS, the strict positivity of $\eta$ is essential, and the sequence $\left\{\xi_{k}\right\}$ plays only a subsidiary role: The sets $\alpha_{0}^{k}$ and $\gamma_{0}^{k}$ should be chosen not too large so that the key condition (3.65) holds as early as possible. To this end, we may choose $\left\{\xi_{k}\right\}$ to be a null sequence. On the other hand, from the computational viewpoint, it is desirable that the set $\beta_{0}^{k}$ is as small as possible, i.e., the sets $\alpha_{0}^{k}$ and $\gamma_{0}^{k}$ are as large as possible. In consequence, it would be important to choose the constant $\eta>0$ and the sequence $\left\{\xi_{k}\right\}$ appropriately.

Another practical choice is simply to remove $\left\{\xi_{k}\right\}$ from Algorithm HIS. For example, we may define

$$
\begin{aligned}
& \alpha_{0}^{k}:=\left\{i \mid \quad G_{i}\left(z^{k}\right)>\eta\right\}, \\
& \gamma_{0}^{k}:=\left\{i \mid \quad H_{i}\left(z^{k}\right)>\eta\right\} \backslash \alpha_{0}^{k}, \\
& \beta_{0}^{k}:=\{1, \cdots, m\} \backslash\left(\alpha_{0}^{k} \cup \gamma_{0}^{k}\right)
\end{aligned}
$$

instead of (3.60)-(3.62), or preferably, let

$$
\begin{aligned}
\alpha_{0}^{k} & :=\left\{i \mid \quad G_{i}\left(z^{k}\right)>\eta, H_{i}\left(z^{k}\right) \leq \eta\right\}, \\
\gamma_{0}^{k} & :=\left\{i \mid \quad G_{i}\left(z^{k}\right) \leq \eta, H_{i}\left(z^{k}\right)>\eta\right\}, \\
\beta_{0}^{k} & :=\{1, \cdots, m\} \backslash\left(\alpha_{0}^{k} \cup \gamma_{0}^{k}\right),
\end{aligned}
$$


which is equivalent to letting $\xi_{k} \equiv \eta(\forall k)$ in (3.60)-(3.62).

Moreover, the parameter $\theta_{k}$ is also expected to be a positive lower bound of (3.53) so that the indices outside $\beta\left(z^{*}\right)$ can be removed from $\beta_{j_{k}}^{k}$ eventually in Step 3. As analyzed in the last subsection, Assumptions A1 and A2 guarantee that the parameter $\theta_{k}$ satisfies this requirement, i.e., $\theta_{k}$ can serve as a positive lower bound of (3.53) when $k$ is large enough.

In a similar way to the last subsection, comprehensive and detailed analysis can be given for Algorithm HIS. In particular, under Assumption A1, $\theta_{k}$ stays at a positive constant when $k$ is sufficiently large in both Cases I and II considered in the last subsection. It then follows from (3.63) that all the indices in $\beta\left(z^{*}\right)$ cannot be selected in Step 3 for all $k$ large enough, i.e., they remain in $\beta_{j_{k}}^{k}$ eventually. Thus, we have the following result.

Theorem 3.9 Suppose that the sequence $\left\{z^{k}\right\}$ generated by Algorithm HIS converges to $z^{*}$ as $\epsilon_{k} \rightarrow 0$ and Assumption A1 holds. Then, for any sufficiently large $k$, we have

$$
\begin{aligned}
& \alpha_{0}^{k} \subseteq \alpha_{1}^{k} \subseteq \cdots \subseteq \alpha_{j_{k}}^{k} \subseteq \alpha\left(z^{*}\right), \\
& \beta_{0}^{k} \supseteq \beta_{1}^{k} \supseteq \cdots \supseteq \beta_{j_{k}}^{k} \supseteq \beta\left(z^{*}\right), \\
& \gamma_{0}^{k} \subseteq \gamma_{1}^{k} \subseteq \cdots \subseteq \gamma_{j_{k}}^{k} \subseteq \gamma\left(z^{*}\right) .
\end{aligned}
$$

If furthermore, Assumption A2 holds, then we have (3.59) for all $k$ sufficiently large.

This theorem indicates that, under similar assumptions to the previous subsection, Algorithm HIS also has a finite termination property.

\section{Further Discussions}

First of all, let us make a remark on Assumption A2 employed by Algorithms HIA and HIS. Recall that, throughout Section 3, the sequence $\left\{z^{k}\right\}$ was assumed to be convergent to $z^{*}$. Actually, the sequence $\left\{z^{k}\right\}$ may have multiple limit points in general. In this case, it is easy to see that, as long as one of the limit points satisfies the assumptions made for $z^{*}$ in Algorithms HIA and HIS, we may obtain similar conclusions. Thus, Assumption A2 can be restated as follows:

$\mathbf{A 2}^{\prime}$ : The set $S$ given in (3.55) contains an accumulation point of the sequence $\left\{z^{k}\right\}$ that is a B-stationary point of problem $(\mathrm{P})$.

This indicates that our assumptions for Algorithms HIA and HIS are really not very restrictive.

\subsection{Stopping criteria}

The stopping criterion in Step 3 of Algorithms H, HIA, and HIS is used to check the Bstationarity of the point $\hat{z}^{k}$, which is based on the fact that, for given $\left(\alpha^{k}, \beta^{k}, \gamma^{k}\right)$, if $\hat{z}^{k}$ is a stationary point of problem (3.6) with nonnegative Lagrange multipliers related to the constraints (3.7) and the MPCC-LICQ holds at $\hat{z}^{k}$, then $\hat{z}^{k}$ is a B-stationary point of problem (P) [11]. Alternatively, we may use some other conditions to check M-stationarity or C-stationarity in view of the fact that, if the MPCC-LICQ holds at $\hat{z}^{k}$ and the Lagrange multipliers $\lambda_{G, i}^{k}$ and $\lambda_{H, i}^{k}$ corresponding to (3.7) satisfy some conditions like (2.7) or (2.8), then $\hat{z}^{k}$ is C- or Mstationary to problem $(\mathrm{P})$. Hence, the algorithms may terminate finitely by producing a $\mathrm{C}$ - or M-stationary point of problem $(\mathrm{P})$ under some weaker conditions. 


\subsection{Comparison of the algorithms}

Comparing with Algorithm H, Algorithms HIA and HIS may need to solve more subproblems (3.32). On the other hand, Algorithm H may have to solve more subproblems $\left(P_{\epsilon}\right)$ than the other two algorithms in general. From both theoretical and computational points of view, problem (3.32) is easier to deal with than problem $\left(P_{\epsilon}\right)$. For example, under the condition that the functions $G, H, h$ are all affine and each $g_{i}$ is convex, the feasible region of problem (3.32) is convex, but that of problem $\left(P_{\epsilon}\right)$ is not convex.

\subsection{Extensions}

In Section 3, we have presented Algorithms H, HIA and HIS by applying an active set identification technique to the smoothing continuation method. Actually, the proposed approaches may be extended by using other subproblems instead of $\left(P_{\epsilon}\right)$ in Step 1 of the algorithms.

(I) Since problem $\left(P_{\epsilon}\right)$ is equivalent to

$$
\begin{array}{cl}
\min & f(z) \\
\text { s.t. } & g(z) \leq 0, h(z)=0 \\
& G(z)+H(z) \geq 0 \\
& G_{i}(z) H_{i}(z)=\epsilon^{2}, i=1, \cdots, m,
\end{array}
$$

we may use problem (4.1) instead of $\left(P_{\epsilon}\right)$ in Step 1 of the algorithms at each iteration. It is obvious that all analysis and conclusions remain valid. Note that, for any $\epsilon>0$, the constraints

$$
G_{i}(z)+H_{i}(z) \geq 0, \quad i=1, \cdots, m
$$

are always inactive and so problem (4.1) seems simpler than $\left(P_{\epsilon}\right)$.

(II) The regularization scheme

$$
\begin{array}{cl}
\min & f(z) \\
\text { s.t. } & g(z) \leq 0, h(z)=0 \\
& G(z) \geq 0, H(z) \geq 0 \\
& G_{i}(z) H_{i}(z) \leq \epsilon, i=1, \cdots, m
\end{array}
$$

and the penalty scheme

$$
\begin{array}{cl}
\min & f(z)+\epsilon^{-1} G(z)^{T} H(z) \\
\text { s.t. } & g(z) \leq 0, h(z)=0 \\
& G(z) \geq 0, H(z) \geq 0,
\end{array}
$$

where $\epsilon$ is a positive parameter, have been proposed as approximate problems of $(\mathrm{P})$ in [22] and [12], respectively. These two methods share similar properties to the smoothing continuation method. We may replace $\left(P_{\epsilon}\right)$ by $(4.2)$ or $(4.3)$ in Step 1 of the algorithms at each iteration and we can obtain similar results. 


\section{Computational Results}

We have tested the proposed algorithms on various instances of MPCCs. In our experiments, we employed the MATLAB 6.0 built-in solver function fmincon to solve the subproblems at each iteration. The computational results indicate that the proposed approach can find an optimal solution of an MPCC in a small number of iterations. We report the details below.

Table 1: Computational results for Problems 5.1-5.3

\begin{tabular}{|c|c|c|c|}
\hline \multirow{5}{*}{ Problem $5.1^{a}$} & \multirow{2}{*}{$\begin{array}{l}\text { Smoothing Con- } \\
\text { tinuation Method }\end{array}$} & $\epsilon_{2}=10^{-6}$ & $(0.5000,0.5000,0.5000,0.5000,-0.0000,-0.0000)$ \\
\hline & & Ite & 27 \\
\hline & \multirow{3}{*}{ Algorithm H } & $\epsilon_{0}=10^{-2}$ & $(0.5000,0.5000,0.5000,0.5000,0,0)$ \\
\hline & & $\beta(\hat{z})$ & $\{1,2\}$ \\
\hline & & Ite & 15 \\
\hline \multirow{5}{*}{ Problem $5.2^{b}$} & \multirow{2}{*}{$\begin{array}{l}\text { Smoothing Con- } \\
\text { tinuation Method }\end{array}$} & $\epsilon_{3}=10^{-8}$ & $(5.0000,4.0000,2.0000,0.0000,0.0000)$ \\
\hline & & Ite & 117 \\
\hline & \multirow{3}{*}{ Algorithm H } & $\epsilon_{0}=10^{-2}$ & $(5.0000,4.0000,2.0000,0.0000,-0.0000)$ \\
\hline & & $\beta(\hat{z})$ & $\emptyset$ \\
\hline & & Ite & 30 \\
\hline \multirow{5}{*}{ Problem $5.3^{c}$} & \multirow{2}{*}{$\begin{array}{l}\text { Smoothing Con- } \\
\text { tinuation Method }\end{array}$} & $\epsilon_{2}=10^{-6}$ & $(25.0000,30.0000,5.0000,10.0000,0.0000)$ \\
\hline & & Ite & 57 \\
\hline & \multirow{3}{*}{ Algorithm H } & $\epsilon_{0}=10^{-2}$ & $(25.0000,30.0000,5.0000,10.0000,0.0000)$ \\
\hline & & $\beta(\hat{z})$ & $\{6\}$ \\
\hline & & Ite & 22 \\
\hline
\end{tabular}

Table 2: Problems 5.4-5.7 generated by QPECgen

\begin{tabular}{c|c|c|c|c}
\hline \multirow{2}{*}{ Parameters $_{\text {in QPECgen }}{ }^{4}$} & \multicolumn{5}{|c}{ Input Data } \\
\cline { 2 - 5 } & Problem 5.4 & Problem 5.5 & Problem 5.6 & Problem 5.7 \\
\hline$(\mathrm{n}, \mathrm{m})$ & $(8,5)$ & $(6,10)$ & $(10,10)$ & $(6,12)$ \\
\hline$(1, \mathrm{p})$ & $(4,5)$ & $(4,10)$ & $(5,10)$ & $(4,12)$ \\
\hline second_deg & 2 & 6 & 5 & 6 \\
\hline first_deg & 2 & 2 & 3 & 2 \\
\hline mix_deg & 2 & 2 & 3 & 4 \\
\hline implicit & 1 & 1 & 0 & 1 \\
\hline
\end{tabular}

$a$ The values of other parameters are common to all problems: qpec_type $=300$, cond_P $=100$, scale $P=100, \quad$ convex $f=1, \quad$ symm $\_=1, \quad$ mono $M=1, \quad$ cond $\_M=200, \quad$ scale $\_M=200, \quad$ tol_deg $=1.0 e-6$, rand_seed $=0$, output $=3$.

\subsection{Computational results for Algorithm $\mathbf{H}$}

In our testing, we set $\epsilon_{0}=10^{-2}$ and updated this parameter by $\epsilon_{k+1}=10^{-2} \epsilon_{k}$. The point $z^{k}$ is used as the starting point for the next step. We employed

$$
\rho_{1}(z)=\|\min (G(z), H(z))\|^{\frac{1}{2}}
$$

and

$$
\rho_{2}(z)=\left\|\Phi_{0}(z)\right\|^{\frac{1}{2}}
$$


Table 3: Some data obtained by QPECgen for Problems 5.4-5.7

\begin{tabular}{c|c|c}
\hline \multirow{3}{*}{ Probelm 5.4 } & $x^{*}$ & $(0.0723,0.4345,0.2387,-0.4003$, \\
& & $-0.2870,-0.5857,-0.4421,0.2286)$ \\
\cline { 2 - 3 } & $y^{*}$ & $(0,0,0,0,0)$ \\
\cline { 2 - 3 } & $\beta\left(x^{*}, y^{*}\right)$ & $\{1,2\}$ \\
\hline \multirow{3}{*}{ Problem 5.5 } & $x^{*}$ & $(0.0872,0.2576,-0.1181,0.2958,-0.1939,-0.0858)$ \\
\cline { 2 - 3 } & $y^{*}$ & $(0,0,0,0,0,0,0,0.7559,0.6660,0.0115)$ \\
\cline { 2 - 3 } & $\beta\left(x^{*}, y^{*}\right)$ & $\{1,2,3,4,5,6\}$ \\
\hline \multirow{3}{*}{ Problem 5.6 } & $x^{*}$ & $(0.6369,0.6371,0.1739,-0.7158,-0.8703$, \\
& & $-0.7478,-0.4383,0.1886,0.0741,0.1494)$ \\
\cline { 2 - 3 } & $y^{*}$ & $(0,0,0,0,0,0,0,0,0,0)$ \\
\cline { 2 - 3 } & $\beta\left(x^{*}, y^{*}\right)$ & $\{1,2,3,4,5\}$ \\
\cline { 2 - 3 } & $x^{*}$ & $(-0.1018,0.0166,-0.3092,0.1948,-0.4273,0.0296)$ \\
\cline { 2 - 3 } & $y^{*}$ & $(0,0,0,0,0,0,0,0,0,0,0.8546,0.3146)$ \\
\hline
\end{tabular}

Table 4: Computational results for Problems 5.4-5.7 ${ }^{a}$

\begin{tabular}{|c|c|c|c|}
\hline \multirow{5}{*}{ Problem 5.4} & \multirow{2}{*}{$\begin{array}{l}\text { Smoothing Con- } \\
\text { tinuation Method }\end{array}$} & $\epsilon_{1}=10^{-4}$ & $(0.0724,0.4345,0.2386,-0.4003,-0.2870)$ \\
\hline & & Ite & 43 \\
\hline & \multirow{3}{*}{ Algorithm H } & $\epsilon_{0}=10^{-2}$ & $(0.0723,0.4345,0.2386,-0.4003,-0.2870)$ \\
\hline & & $\beta(\hat{x}, \hat{y})$ & $\{1,2\}$ \\
\hline & & Ite & 32 \\
\hline \multirow{5}{*}{ Problem 5.5} & \multirow{2}{*}{$\begin{array}{l}\text { Smoothing Con- } \\
\text { tinuation Method }\end{array}$} & $\epsilon_{2}=10^{-6}$ & $(0.0872,0.2576,-0.1181,0.2958,-0.1940)$ \\
\hline & & Ite & 103 \\
\hline & \multirow{3}{*}{ Algorithm H } & $\epsilon_{1}=10^{-4}$ & $(0.0871,0.2577,-0.1181,0.2957,-0.1940)$ \\
\hline & & $\beta(\hat{x}, \hat{y})$ & $\{1,2,3,4,5,6\}$ \\
\hline & & Ite & 59 \\
\hline \multirow{5}{*}{ Problem 5.6} & \multirow{2}{*}{$\begin{array}{l}\text { Smoothing Con- } \\
\text { tinuation Method }\end{array}$} & $\epsilon_{3}=10^{-8}$ & $(0.6370,0.6366,0.1738,-0.7158,-0.8703)$ \\
\hline & & Ite & 185 \\
\hline & \multirow{3}{*}{ Algorithm H } & $\epsilon_{1}=10^{-4}$ & $(0.6368,0.6371,0.1739,-0.7158,-0.8704)$ \\
\hline & & $\beta(\hat{x}, \hat{y})$ & $\{1,2,3,4,5\}$ \\
\hline & & Ite & 132 \\
\hline \multirow{5}{*}{ Problem 5.7} & \multirow{2}{*}{$\begin{array}{l}\text { Smoothing Con- } \\
\text { tinuation Method }\end{array}$} & $\epsilon_{3}=10^{-8}$ & $(-0.1002,0.0159,-0.3091,0.1947,-0.4276)$ \\
\hline & & Ite & 104 \\
\hline & \multirow{3}{*}{ Algorithm H } & $\epsilon_{1}=10^{-4}$ & $(-0.1018,0.0167,-0.3091,0.1947,-0.4275)$ \\
\hline & & $\beta(\hat{x}, \hat{y})$ & $\{1,2,3,4,5,6\}$ \\
\hline & & Ite & 41 \\
\hline
\end{tabular}

${ }^{a}$ Initial points are $(0.5,0.5, \cdots, 0.5)$ for Problems $5.4,5.5$ and 5.7 , and $(2,2, \cdots, 2)$ for Problem 5.6.

as the identification function $\rho$ in Step 1 of Algorithm $\mathrm{H}$, and we found that the two functions yielded almost the same numerical results for all examples solved. This is not surprising because $\left\{\rho_{1}\left(z^{k}\right)\right\}$ and $\left\{\rho_{2}\left(z^{k}\right)\right\}$ tend to 0 in the same order as $z^{k} \rightarrow z^{*}$.

First, we report on numerical results for problems from an AMPL collection of MPECs called MacMPEC [15]. We notice that, since most problems are small-scale and, especially, the cardinalities of the lower-level degenerate index sets at the solutions are quite low, the proposed approach always solved the problems in only one iteration. Here, we only show the results for three problems, say Problems 5.1, 5.2, and 5.3, which are coded as desilva.mod, ex.9.1.1.mod, and bilevel1.mod, respectively, in MacMPEC. The computational results with the identification function $\rho_{1}$ are reported in Table 1, where $\hat{z}$ denotes the points obtained by Algorithm $\mathrm{H}, z^{*}$ and $z^{0}$ indicate the solution of the problem and the initial point employed in the testing. In addition, $\beta\left(z^{*}\right)$ denotes the lower-level degenerate index set estimated at the point $z^{*}$ and Ite stands for the number of total iterations spent by the solver fmincon. Recall that the objective of the hybrid approach is to identify the set $\beta\left(z^{*}\right)$. 
Note that, in Table 1, we only list the values of the first five components of the variables because that would be sufficient to illustrate the behavior of the tested algorithms. Table 1 shows that Algorithm $\mathrm{H}$ was able to find the solutions of Problems 5.1, 5.2, and 5.3 very quickly. This, as we mentioned above, may be due to the small scale of the problems and the low cardinality of the lower-level degenerate index sets at the solutions.

Next we report on numerical results for somewhat larger test problems generated by QPECgen of Jiang and Ralph [14]. The QPECgen generator is a MATLAB program that uses a set of parameters, see [14] for detail. Once these parameters are specified, the program can randomly generate a quadratic program with linear complementarity constraints

$$
\begin{array}{ll}
\min & \frac{1}{2}\left(x^{T}, y^{T}\right) P\left(\begin{array}{l}
x \\
y
\end{array}\right)+c^{T} x+d^{T} y \\
\text { s.t. } & A\left(\begin{array}{l}
x \\
y
\end{array}\right)+a \leq 0, \\
& y \geq 0, N x+M y+q \geq 0, \\
& y^{T}(N x+M y+q)=0,
\end{array}
$$

where $P, A, N, M$ are constant matrices and $c, d, a, q$ are constant vectors with appropriate dimensions. QPECgen also outputs an approximate solution of a generated problem.

We set the QPECgen parameters as in Table 2 to generate Problems 5.4-5.7. In particular, the parameters $\mathrm{n}$ and $\mathrm{m}$ denote the dimensions of the variables $x$ and $y$, respectively, and second_deg stands for the cardinality of the lower-level degenerate index set at a solution.

Some data obtained by the QPECgen generator are summarized in Table 3 , in which $\left(x^{*}, y^{*}\right)$ denotes the solution given by QPECgen and $\beta\left(x^{*}, y^{*}\right)$ stands for the lower-level degenerate index set at $\left(x^{*}, y^{*}\right)$, i.e., $\beta\left(x^{*}, y^{*}\right):=\left\{i \mid\left(N x^{*}+M y^{*}+q\right)_{i}=0=y_{i}^{*}\right\}$. The computational results with the identification function $\rho_{1}$ for Problems 5.4-5.7 are reported in Table 4, where the values of the first five components of the variables are displayed for each of the obtained solutions.

The results shown in the tables reveal that it was not difficult to identify the active sets by Algorithm $\mathrm{H}$, at least for the test problems used in our numerical experiments, although we have observed that the penalty method may not be very stable when the parameter $\epsilon$ becomes small. In fact, we got the correct active sets in no more than three steps in all cases and, since the computed points satisfy the B-stationarity conditions, Algorithm H terminated. Especially, as mentioned in the last section, Algorithm $\mathrm{H}$ may terminate by finding a solution before the correct index sets are obtained, see Table 1. Moreover, we notice that, since the number of active indices is larger than the dimension of $z$, Problems 5.2, 5.5, and 5.7 do not satisfy the MPCC-LICQ at the solutions. Nevertheless, we were able to obtain the solutions successfully, which shows the robustness of the proposed approach.

Table 5: Parameters in QPECgen for Problems 5.8-5.9

\begin{tabular}{cccccccccc}
\hline Problem & qpec_type & $(\mathrm{n}, \mathrm{m})$ & $(\mathrm{l}, \mathrm{p})$ & cond_P & scale_P & convex_f & symm_M & mono_M & cond_M \\
\hline$\# 5.8$ & 300 & $(10,10)$ & $(5,10)$ & 100 & 100 & 1 & 1 & 1 \\
$\# 5.9$ & 300 & $(8,14)$ & $(4,14)$ & 100 & 100 & 1 & 1 & 1 \\
\hline Problem & scale_M & second_deg & first_deg & mix_deg & tol_deg & implicit & rand_seed & output \\
\hline \# 5.8 & 200 & 4 & 3 & 3 & $1.0 \mathrm{e}-6$ & 0 & 0 & 3 \\
\hline 5.9 & 200 & 4 & 2 & 1 & $1.0 \mathrm{e}-6$ & 0 & 0 & 3 \\
\hline
\end{tabular}


Table 6: Some data obtained by QPECgen for Problems 5.8-5.9

\begin{tabular}{cccc}
\hline Problem & $x^{*}$ & $y^{*}$ & $\beta\left(x^{*}, y^{*}\right)$ \\
\hline$\#$ 5.8 & $(0.6369,0.6371,0.1739,-0.7158,-0.8703$, & $(0,0, \cdots, 0)$ & $\{1,2,3,4\}$ \\
& $-0.7478,-0.4383,0.1886,0.0741,0.1494)$ & & \\
\hline$\# 5.9$ & $(-0.7330,-0.2090,-0.4140,0.0168$, & $(0, \cdots, 0,0.4681,0.4739,0.2088)$ & $\{1,2,3,4\}$ \\
& $-0.7084,-0.1104,0.0030,-0.4658)$ & & \\
\hline
\end{tabular}

Table 7: Computational results for Problem $5.8^{a}$

\begin{tabular}{c|c|lcc}
\hline & $\epsilon_{k}$ & degenerate set & distance & Ite \\
\hline \multirow{3}{*}{ Algorithm H } & $10^{-2}$ & $\beta^{k}=\emptyset$ & 0.0007 & $25+10$ \\
\cline { 2 - 5 } & $10^{-3}$ & $\beta^{k}=\emptyset$ & 0.0010 & $29+10$ \\
\cline { 2 - 5 } & $10^{-4}$ & $\beta^{k}=\{4\}$ & 0.0005 & $23+11$ \\
\hline & & $\beta_{0}^{k}=\emptyset$ & 0.0007 & $25+10$ \\
Algorithm HIA & \multirow{2}{*}{$10^{-2}$} & $\beta_{1}^{k}=\{4\}$ & 0.0007 & 10 \\
& & $\beta_{2}^{k}=\{2,4\}$ & 0.0005 & 11 \\
& & $\beta_{3}^{k}=\{1,2,4\}$ & 0.0004 & 10 \\
& & $\beta_{4}^{k}=\{1,2,3,4\}$ & 0.0004 & 10 \\
\hline \multirow{3}{*}{ Algorithm HIS } & \multirow{2}{*}{$10^{-2}$} & $\beta_{0}^{k}=\{1,2,3,4,7,9,10\}$ & 1.5944 & $25+6$ \\
& & $\beta_{1}^{k}=\{1,2,3,4,9,10\}$ & 1.2922 & 5 \\
& & $\beta_{2}^{k}=\{1,2,3,4,10\}$ & 0.4131 & 9 \\
& & $\beta_{3}^{k}=\{1,2,3,4\}$ & 0.0004 & 10 \\
\hline
\end{tabular}

${ }^{a}$ We used $(1,1, \cdots, 1)$ as the initial point for all methods and we set $\theta_{0}=0.2$ in Algorithms HIA and HIS. In addition, the parameter $\eta$ in Algorithm HIS is set to be 0.5.

\subsection{Computational results for Algorithms HIA and HIS}

In this subsection, we examine the effectiveness of Algorithms HIA and HIS on some examples of MPCC. Since the numerical results shown in the last subsection have revealed that Algorithm $\mathrm{H}$ is comparable to the smoothing continuation method [10], we only compare Algorithms HIA and HIS with Algorithm H. ${ }^{1}$

We show the QPECgen parameters used to generate Problems 5.8 and 5.9 in Table 5, and summarize some data output by the QPECgen generator in Table 6 . In our experiments, we set $\epsilon_{0}=10^{-2}$ and updated this parameter by $\epsilon_{k+1}=10^{-1} \epsilon_{k}$. Moreover, we employ

$$
\rho(x, y)=\|\min (N x+M y+q, y)\|^{\frac{4}{5}}
$$

as the identification function in Step 1 of both Algorithm H and Algorithm HIA. In Algorithm HIS, we use the sequence $\left\{\xi_{k}\right\}$ given by

$$
\xi_{k}=\rho\left(x^{k}, y^{k}\right), \quad k=0,1, \cdots,
$$

which is a reasonable choice to compare with the other two methods. See the tables for the setting of the other parameters involved.

The computational results for Problem 5.8 and 5.9 are reported in Tables 7 and 8, respectively. In the tables, distance denotes the distance between the obtained point and the solution $\left(x^{*}, y^{*}\right)$ measured by the infinity norm. In the Ite column, a sum $\nu_{1}+\nu_{2}$ means that $\nu_{1}$ is the number of iterations spent by fmincon for solving problem $\left(\mathrm{P}_{\epsilon}\right)$ and $\nu_{2}$ denotes the number of iterations spent by solving subproblem (3.32), whereas a single number $\nu$ stands for the number of iterations spent by solving subproblem (3.32) (as there is no need to solve problem $\left(\mathrm{P}_{\epsilon}\right)$ in these cases).

\footnotetext{
${ }^{1}$ We have also confirmed through numerical experiments, the details of which are not included in this paper, that Algorithm $\mathrm{H}$ is also comparable to other existing methods such as the penalty function method [12] and the regularization method [22].
} 
Table 8: Computational results for Problem $5.9^{a}$

\begin{tabular}{c|c|lcc}
\hline & $\epsilon_{k}$ & degenerate set & distance & Ite \\
\hline \multirow{3}{*}{ Algorithm H } & $10^{-2}$ & $\beta^{k}=\emptyset$ & 0.0003 & $27+6$ \\
\cline { 2 - 5 } & $10^{-3}$ & $\beta^{k}=\{1,2,3\}$ & 0.0004 & $19+5$ \\
\cline { 2 - 5 } & $10^{-4}$ & $\beta^{k}=\{1,2,3,4\}$ & 0.0003 & $35+7$ \\
\hline & & $\beta_{0}^{k}=\emptyset$ & 0.0003 & $27+6$ \\
Algorithm HIA & \multirow{2}{*}{$10^{-2}$} & $\beta_{1}^{k}=\{2\}$ & 0.0003 & 6 \\
& & $\beta_{2}^{k}=\{1,2\}$ & 0.0009 & 3 \\
& & $\beta_{3}^{k}=\{1,2,3\}$ & 0.0009 & 3 \\
& & $\beta_{4}^{k}=\{1,2,3,4\}$ & 0.0009 & 3 \\
\hline \multirow{3}{*}{ Algorithm HIS } & \multirow{2}{*}{$10^{-2}$} & $\beta_{0}^{k}=\{1,2,3,4,11\}$ & 0.8554 & $27+6$ \\
\hline
\end{tabular}

${ }^{a}$ We employed $(1,1, \cdots, 1)$ as the initial point for all methods. We set $\theta_{0}=0.2$ in Algorithms HIA and HIS and, $\eta=0.2$ in Algorithm HIS.

The results shown in the tables reveal that both Algorithms HIA and HIS were able to identify the active sets successfully. As mentioned in the previous section, Algorithms HIA and HIS need to solve more problems of the form (3.32) than Algorithm H, whereas the latter has to solve more problems of the form $\left(P_{\epsilon}\right)$. Our experiments show that problem (3.32) can be solved in fewer iterations than problem $\left(P_{\epsilon}\right)$ generally.

\section{Conclusions}

By incorporating an active set identification technique with the smoothing continuation method, we have presented three hybrid algorithms for MPCC. Unlike most of the existing methods, the proposed algorithms possess a finite termination property, that is, we may compute a point with some kind of stationarity to problem $(\mathrm{P})$ by solving a finite number of nonlinear programs. In consequence, the proposed approach is appealing for solving MPCC.

In particular, Algorithms $\mathrm{H}$ and HIA are based on an identification function satisfying (3.9)(3.11). Appropriate identification functions may improve efficiency of the two algorithms. Under the assumption of the asymptotically weak nondegeneracy of the sequence $\left\{z^{k}\right\}$ generated in Step 1 of the algorithms, we have given two identification functions in Theorems 3.3 and 3.4. It is still an open question to find a new identification function that works under weaker conditions.

Acknowledgement. The authors are grateful to Professor Paul Tseng for his helpful suggestions on an earlier version of the paper.

\section{References}

[1] J.V. Burke, On the identification of active constraints II: The nonconvex case, SIAM Journal on Numerical Analysis, 27 (1990), 1081-1102.

[2] J.V. Burke And J.J. Moré, On the identification of active constraints, SIAM Journal on Numerical Analysis, 25 (1988), 1197-1211.

[3] X. Chen and M. Fukushima, A smoothing method for a mathematical program with Pmatrix linear complementarity constraints, Computational Optimization and Applications, 27 (2004), 223-246. 
[4] Y. Chen And M. Florian, The nonlinear bilevel programming problem: Formulations, regularity and optimality conditions, Optimization, 32 (1995), 193-209.

[5] F. Facchinei, A. Fischer, And C. Kanzow, On the accurate identification of active constraints, SIAM Journal on Optimization, 9 (1998), 14-32.

[6] F. Facchinei, A. Fischer, And C. Kanzow, On the identification of zero variables in an interior-point framework, SIAM Journal on Optimization, 10 (2000), 1058-1078.

[7] F. FACChIneI, H. Jiang, AND L. QI, A smoothing method for mathematical programs with equilibrium constraints, Mathematical Programming, 85 (1999), 107-134.

[8] C.A. Floudas, P.M. Pardalos, C.S. Adjiman, W.R. Esposito, Z.H. Gümüs, S.T. Harding, J.L. Klepeis, C.A. Meyer and C.A. Schweiger, Handbook of Test Problems in Local and Global Optimization, Kluwer Academic Publishers, Dordrecht, 1999.

[9] M. Fukushima, Z.Q. Luo, And J.S. PAng, A globally convergent sequential quadratic programming algorithm for mathematical programs with linear complementarity constraints, Computational Optimization and Applications, 10 (1998), 5-34.

[10] M. Fukushima AND J.S. PANG, Convergence of a smoothing continuation method for mathematical problems with complementarity constraints, Ill-posed Variational Problems and Regularization Techniques, Lecture Notes in Economics and Mathematical Systems, Vol. 477, M. Théra and R. Tichatschke (eds.), Springer-Verlag, Berlin/Heidelberg, 1999, $105-116$.

[11] M. Fukushima And P. Tseng, An implementable active-set algorithm for computing a B-stationary point of the mathematical program with linear complementarity constraints, SIAM Journal on Optimization, 12 (2002), 724-739.

[12] X. Hu AND D. RALPH, Convergence of a penalty method for mathematical programming with equilibrium constraints, Journal of Optimization Theory and Applications, to appear.

[13] X.X. Huang, X.Q. YAng, And D.L. Zhu, A sequential smooth penalization approach to mathematical programs with complementarity constraints, manuscript, Department of Applied Mathematics, Hong Kong Polytechnic University, Hong Kong, 2001.

[14] H. JiANG AND D. RALPH, QPECgen, a MATLAB generator for mathematical programs with quadratic objectives and affine variational inequality constraints, Computational Optimization and Applications, 13 (1999), 25-59.

[15] S. LEYFFeR, MacMPEC: AMPL collection of MPECs, Technical Report, http://www-unix.mcs.anl.gov/ leyffer/MacMPEC/, 2000.

[16] H. JiAng AND D. RALPH, Smooth SQP methods for mathematical programs with nonlinear complementarity constraints, SIAM Journal on Optimization, 10 (2000), 779-808.

[17] G.H. Lin And M. Fukushima, A modified relaxation scheme for mathematical programs with complementarity constraints, Annals of Operations Research, to appear.

[18] G.H. Lin And M. Fukushima, A new relaxation method for mathematical programs with complementarity constraints, Journal of Optimization Theory and Applications, 118 (2003), 81-116. 
[19] G.H. Lin And M. Fukushima, Some exact penalty results for nonlinear programs and their applications to mathematical programs with equilibrium constraints, Journal of Optimization Theory and Applications, 118 (2003), 67-80.

[20] Z.Q. Luo, J.S. PAng, and D. Ralph, Mathematical Programs with Equilibrium Constraints, Cambridge University Press, Cambridge, United Kingdom, 1996.

[21] H. Scheel And S. Scholtes, Mathematical programs with complementarity constraints: Stationarity, optimality, and sensitivity, Mathematics of Operations Research, 25 (2000), $1-22$.

[22] S. Scholtes, Convergence properties of a regularization scheme for mathematical programs with complementarity constraints, SIAM Journal on Optimization, 11 (2001), 918-936.

[23] S. Scholtes AND M. StÖHR, How stringent is the linear independence assumption for mathematical programs with complementarity constraints, Mathematics of Operations Research, 26 (2001), 851-863.

[24] P. Tseng, Growth behavior of a class of merit functions for the nonlinear complementarity problem, Journal of Optimization Theory and Applications, 89 (1996), 17-37.

[25] N. Yamashita, H. Dan, And M. Fukushima, On the identification of degenerate indices in the nonlinear complementarity problem with the proximal point algorithm, Mathematical Programming, 99 (2004), 377-397. 